\title{
4 Spectral dependence of MODIS cloud droplet effective radius retrievals for marine boundary layer clouds
}

Zhibo Zhang, Steven Platnick, Andrew S. Ackerman, and Hyoun-Myoung Cho

\subsection{Introduction}

Low-level warm marine boundary layer (MBL) clouds cover large regions of Earth's surface. They have a significant role in Earth's radiative energy balance (Klein and Hartmann, 1993) and hydrological cycle. Despite the fundamental role of low-level warm water clouds in climate, our understanding of these clouds is still limited. In particular, connections between their properties (e.g. cloud fraction, cloud water path, and cloud droplet size) and environmental factors such as aerosol loading and meteorological conditions continue to be uncertain or unknown. Modeling these clouds in climate models remains a challenging problem. As a result, the influence of aerosols on these clouds in the past and future, and the potential impacts of these clouds on global warming remain open questions leading to substantial uncertainty in climate projections. To improve our understanding of these clouds, we need continuous observations of cloud properties on both a global scale and over a long enough timescale for climate studies. At present, satellite-based remote sensing is the only means of providing such observations.

The cloud droplet effective radius $\left(r_{e}\right)$ is one of the most important cloud parameters that are routinely monitored from space. The $r_{e}$ is defined as (Hansen and Travis, 1974)

$$
r_{e}(z)=\frac{\int_{0}^{\infty} r^{3} n(r, z) d r}{\int_{0}^{\infty} r^{2} n(r, z) d r},
$$

where $n(r, z)$ is the cloud droplet size distribution (DSD) at altitude $z$ in cloud. The cloud droplet effective radius determines the optical thickness of cloud for a given amount of water (Twomey, 1974). It also has a significant influence on precipitation formation processes (Lebsock et al., 2008; Kubar et al., 2009). Therefore, it is a key microphysical parameter required to estimate radiative effects of clouds, study aerosol-cloud-precipitation interactions, and validate cloud parameterization in global climate models.

Many remote sensing methods exist to infer cloud $r_{e}$ from various types of satellite instruments (e.g. Prabhakara et al., 1988; Nakajima and King, 1990; Austin et al., 2009). Of particular interest in this review are cloud $r_{e}$ retrievals from MODIS (or Moderate Resolution Imaging Spectroradiometer). MODIS is a key instrument 
aboard the Terra and Aqua satellites. Terra's orbit around Earth is timed so that it passes from north to south across the equator in the morning, while Aqua passes south to north over the equator in the afternoon. Terra and Aqua MODIS are viewing the entire Earth's surface every one to two days, acquiring data in 36 spectral bands. A variety of cloud parameters, from cloud fraction to cloud top height to cloud optical thickness $(\tau)$ and $r_{e}$, can be inferred from MODIS multispectral observations (Platnick et al., 2003). The operational MODIS cloud retrieval algorithm, as described in detail in the next section, is based on the so-called bi-spectral solar reflective method (referred to as bi-spectral method hereafter), which utilizes cloud reflectance measurements from two spectral bands to retrieve cloud $\tau$ and $r_{e}$, simultaneously (Nakajima and King, 1990). One measurement is usually made in the visible or near-infrared spectral region (e.g. $0.86 \mu \mathrm{m}$ ), where water absorption is negligible and therefore cloud reflection is mainly determined by $\tau$, and the other in the shortwave infrared (SWIR) (e.g. $2.1 \mu \mathrm{m}$ or $3.7 \mu \mathrm{m}$ ), where water is significantly absorptive and cloud reflectance primarily decreases with increasing cloud droplet size. The $\tau$ and $r_{e}$ retrievals based on the bi-spectral method are widely used for validating climate models (Kay et al., 2012; Pincus et al., 2012), studying aerosol-cloud interactions (Quaas and Boucher, 2005; Quaas et al., 2009) and facilitating other cloud remote sensing techniques (Lebsock and L'Ecuyer, 2011).

Unlike cloud optical properties (such as $\tau$ ) that can be spectrally dependent, cloud $r_{e}$, a physical cloud parameter, should be independent of the observation method or what spectral band is used. For example, the MODIS instrument has three SWIR bands, centered at $1.6 \mu \mathrm{m}, 2.1 \mu \mathrm{m}$, and $3.7 \mu \mathrm{m}$, respectively. In the MODIS operational cloud product (MOD06) Collection 5 (C5) processing algorithm, the combination of the 0.86 and $2.1 \mu \mathrm{m}$ bands are used for $\tau$ and $r_{e}$ retrievals over open ocean (Platnick et al., 2003). Hereafter, we will refer to the $r_{e}$ retrieval based on the 2.1- $\mu \mathrm{m}$ band observation as $r_{e, 2.1}$. Besides $r_{e, 2.1}$, the MODIS also provides two other $r_{e}$ retrievals, one based on the 1.6- $\mu \mathrm{m}$ band and the other based on the $3.7-\mu \mathrm{m}$ band observations (hereafter referred to as $r_{e, 1.6}$ and $r_{e, 3.7}$ ). One might expect these three $r_{e}$ retrievals to be in close agreement. However, several studies have found substantial differences between them (Nakajima et al., 2010a; Seethala and Horváth, 2010; Zhang and Platnick, 2011). In particular, it is found that:

- MODIS $r_{e, 3.7}$ retrievals for marine warm clouds are generally smaller than $r_{e, 2.1}$ (and $r_{e, 1.6}$ );

- Geographically, the differences between $r_{e, 3.7}$ and $r_{e, 2.1}$ based on MODIS Level 3 monthly mean product show strong dependence on cloud regimes, small variations (close to zero) over the costal stratocumulus regimes, and large variations over the cumulus cloud regimes;

- At pixel level, the difference between $r_{e, 3.7}$ and $r_{e, 2.1}$ correlates with cloud $\tau$, $r_{e}$, and the degree of sub-pixel inhomogeneity.

A detailed analysis of the differences between $r_{e, 3.7}$ and $r_{e, 2.1}$ MODIS retrievals will be given in section 4.3. $r_{e, 1.6}$ is not considered in this study, mainly because the 1.6- $\mu \mathrm{m}$ band on Aqua MODIS has nonfunctional or noisy detectors that lead to the 
striping issue (Wang et al., 2006). As a result, it is difficult to make pixel-to-pixel comparisons between $r_{e, 1.6}$ and other effective radius retrievals.

The spectral dependence of MODIS $r_{e}$ raises many questions. The most important one, for all the users of MODIS products, is whether the difference between $r_{e, 3.7}$ and $r_{e, 2.1}$ is an artifact due to, say, algorithm issues and inherent limitations of the retrieval method, or something meaningful that contains information about the cloud. Several lines of evidence suggest that it is unlikely to be due to technical issues (e.g. code bugs, ancillary data issues, etc.) of the operational MODIS cloud retrieval algorithm. First, several studies based on independent retrieval algorithms also found substantial differences between $r_{e, 3.7}$ and $r_{e, 2.1}$ that are similar to the operational MODIS cloud product (Nakajima et al., 2010a; Minnis et al., 2011). Second, both theoretical and numerical studies indicate that $r_{e, 3.7}$ and $r_{e, 2.1}$ are expected to differ significantly under certain circumstances owing to their difference sensitivities to, for example, cloud vertical structure (Platnick, 2000), 3D radiative effects (Zhang and Platnick, 2011; Zhang et al., 2012), and the presence of drizzle drops in the cloud (Nakajima and King, 1990; Zhang, 2013).

The question is then: what has caused the differences between $r_{e, 3.7}$ and $r_{e, 2.1}$ ? A number of recent studies have attempted to address this question from different perspectives (Platnick, 2000; Nakajima et al., 2010a, 2010b; Seethala and Horváth, 2010; Zhang et al., 2010, 2012; Zinner et al., 2010; Zhang and Platnick, 2011; Zhang, 2013). Several mechanisms have been proposed to explain the abovementioned spectral dependence of MODIS $r_{e}$ retrieval, which can be divided into two categories based on their underlying physics and consequent implications. In the first category are those mechanisms related to the fact that $r_{e, 3.7}$ and $r_{e, 2.1}$ have different sensitivities to cloud vertical structure and the presence of large drizzle drops in warm water clouds, with implications that the spectral difference actually carries useful information about the cloud that can be used for remote sensing and model validation. In the other category are those mechanisms related to the inherent limitations of the bi-spectral method, such as, the lack of consideration of $3 \mathrm{D}$ radiative effects and sub-pixel inhomogeneity in the retrieval method. In such cases, the spectral difference implies significant retrieval uncertainties should be cautioned when using MODIS $r_{e}$ retrieval products. Overall, these studies reveal that the spectral difference of MODIS $r_{e}$ retrievals is a complicated issue that has complex causes.

This chapter provides an overview of the current understanding of the spectral dependence of MODIS $r_{e}$ retrievals. In section 4.2 , we briefly revisit the operational MODIS cloud $r_{e}$ retrieval algorithm to set the stage for later discussion. In section 4.3, the MODIS $r_{e, 3.7}$ and $r_{e, 2.1}$ retrievals for MBL clouds are compared to illustrate the spectral dependence of MODIS cloud $r_{e}$ retrieval. Section 4.4 introduces several potential mechanisms that may cause or contribute to the spectral dependence of MODIS $r_{e}$ retrieval. The relative role of these mechanisms in different cloud regimes is discussed in section 4.5. Current outstanding issues and an overview for future work are given in section 4.6. 


\subsection{Operational MODIS $r_{e}$ retrieval algorithm}

In order to set the stage for later discussion in this section, we briefly describe the operational MODIS $r_{e}$ and $\tau$ retrieval algorithm based on the bi-spectral method. The retrieval makes use of a pair of measurements of cloud reflectivity, one from a visible or near-infrared (VNIR) MODIS band (0.86- $\mu \mathrm{m}$ band over ocean) and the other from a shortwave infrared (SWIR) band (e.g. $2.1 \mu \mathrm{m}$ or $3.7 \mu \mathrm{m}$ band) (Nakajima and King, 1990; Platnick et al., 2003). The visible band measurement provides the information for $\tau$ retrieval because water absorption in the VNIR region is almost negligible and, as a result, cloud reflectivity is mainly determined by $\tau$. The SWIR measurement provides the information for $r_{e}$ retrieval because significant water absorption in SWIR makes cloud reflectance decrease with increasing cloud $r_{e}$. In the operational algorithm, this method is implemented by using the so-called look-up-table (LUT), as shown in Fig. 4.1. The LUT contains pre-computed cloud reflectivities at visible and SWIR bands for various combinations of $r_{e}$ and $\tau$ under different Sun-satellite viewing geometries and surface reflectances. As illustrated in Fig. 4.1, in practice. $r_{e}$ and $\tau$ are retrieved by projecting the observed reflectivities onto the LUT. Once $r_{e}$ and $\tau$ are retrieved, the liquid water path (LWP) of the cloud can be easily derived from the equation $L W P=\frac{2}{3} \rho \tau r_{e}$ assuming that the cloud layer is vertically homogenous and the extinction coefficient $Q_{e}$ of a cloud droplet is about 2. The MODIS cloud $r_{e}$ and $\tau$ retrievals have been shown to agree reasonably well with other satellite products (e.g. Zhang et al., 2009; Minnis et al., 2011; Pincus et al., 2012; Stubenrauch et al., 2012; Walther and Heidinger, 2012) and in situ measurements (e.g. Painemal and Zuidema, 2011; King et al., 2012).

a) $\boldsymbol{R}(0.86 \mu \mathrm{m}) \& \boldsymbol{R}(2.1 \mu \mathrm{m})$ LUT

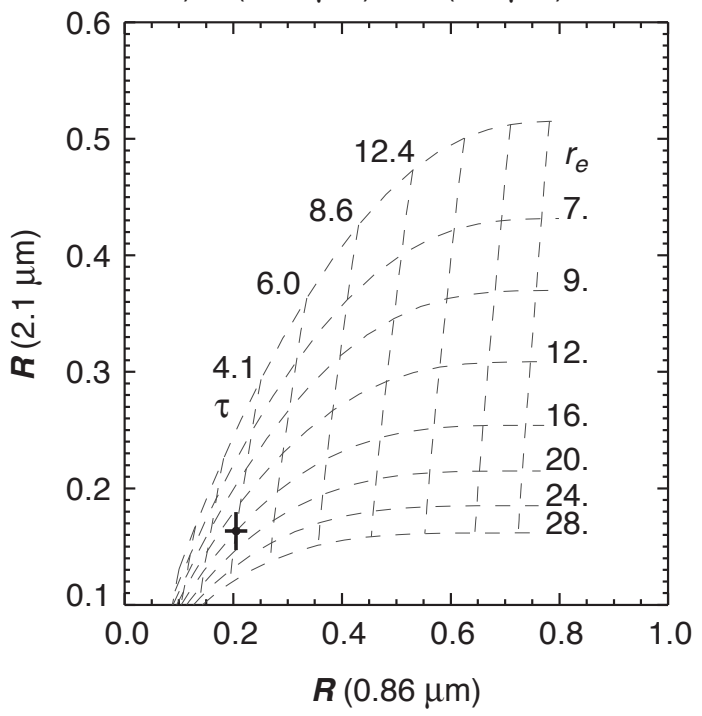

b) $\boldsymbol{R}(0.86 \mu \mathrm{m}) \& \boldsymbol{R}(3.7 \mu \mathrm{m})$ LUT

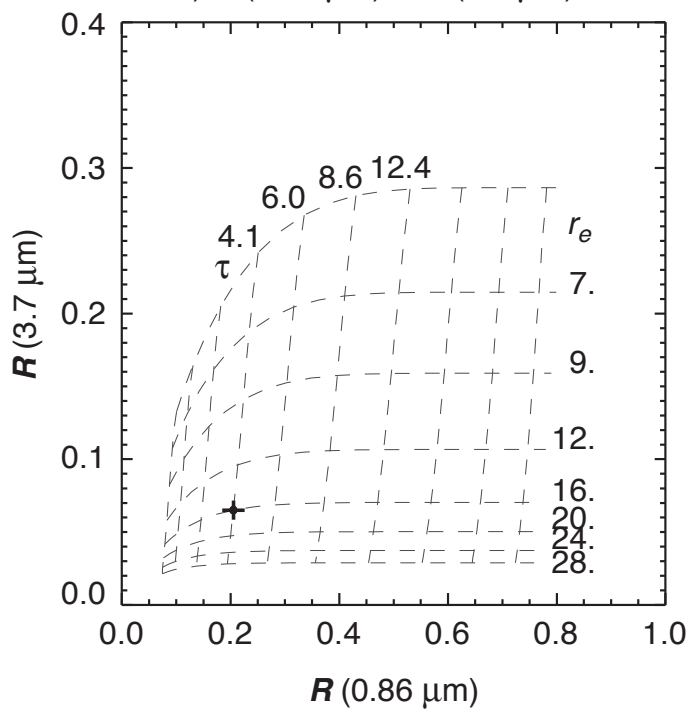

Fig. 4.1. Examples of the look-up-table of cloud bi-directional reflection function as functions of cloud optical thickness and effective radius, based on the combination of (a) 0.86 and $2.1-\mu \mathrm{m}$ bands, and (b) 0.86 and $3.7-\mu \mathrm{m}$ bands. 
It is important to note that the bi-spectral method is based on several important assumptions about the cloud (or the cloudy pixel):

1. Within the pixel, the cloud is vertically homogeneous. (referred to as the vertical homogenous assumption).

2. Within the pixel, the cloud is horizontally homogenous. The cloud reflectance of the pixel of interest is independent of the properties of surrounding pixels. (referred to as the plane-parallel and homogeneous cloud assumption).

3. Within the pixel, the sizes of cloud droplets follow certain analytical distributions, namely the following monomodal Gamma distribution (King et al., 1998):

$$
n(r)=N r^{\frac{1-3 v_{e}}{v_{e}}} \exp \left(-\frac{1}{v_{e}} \frac{r}{r_{e}}\right)
$$

where $n(r)$ is DSD, $N$ is a constant, and $v_{e}$ is the effective variance assumed to be 0.1 in the operational MODIS algorithm (monomodal Gamma DSD assumption hereafter).

These assumptions are necessary because, at most, two independent pieces of information can be extracted from a pair of cloud reflectivities. Since the information content has been used for $r_{e}$ and $\tau$ retrievals, other aspects of cloud would have to be assumed. These assumptions are justified for some clouds, particularly, nonprecipitating stratocumulus clouds (Martin et al., 1994; Di Girolamo et al., 2010). However, for other clouds, they can be problematic. For example, it is known that trade wind cumulus clouds can be far from plane-parallel (Liang et al., 2009; Di Girolamo et al., 2010). The vertical homogeneous assumption and the monomodal Gamma DSD often break down when precipitation begins to form within the cloud. The warm rain processes, such as collision-coalescence, could broaden the DSD and sometimes give rise to a second mode, the so-called drizzle or rain mode, leading to a bi-modal DSD (Berry, 1967; Berry and Reinhardt, 1974; Pruppacher and Klett, 1997). In addition, the collision-coalescence processes make drizzle drops grow bigger as they fall from cloud top towards cloud base, inducing vertical structures within the cloud (Pruppacher and Klett, 1997). When these conditions occur and the above-mentioned assumptions break down, MODIS $r_{e}$ and $\tau$ retrievals face substantial uncertainties. It will be shown later in section 4.4 that the mechanisms that cause significant differences between $r_{e, 3.7}$ and $r_{e, 2.1}$ are more or less connected to breakdown of these fundamental assumptions about cloud made in MODIS retrieval.

\subsection{Spectral dependence of MODIS $r_{e}$ retrievals for MBL clouds}

As stated from the beginning, we focus only on the warm liquid-phase clouds over ocean. Since such clouds reside mostly in the MBL, we will refer to them as MBL clouds for simplicity. We attempt to identify a MODIS pixel as MBL cloud based on the following criteria: (i) the pixel is labeled as 'confident cloudy' by the 1-km MODIS cloud mask product (MOD35); (ii) over ocean; (iii) labeled as 'liquid water' 
by the MODIS 1-km 'Cloud_Phase_Optical_Properties' data set within MOD06; (iv) cloud top temperature warmer than $273 \mathrm{~K}$. These conditions are expected to remove most of the situations that may complicate the analyses, such as thin cirrus overlapping lower clouds.

\subsubsection{Geographical pattern}

The monthly mean $r_{e, 2.1}$ and $r_{e, 3.7}$ for MBL clouds based on May 2007 MODIS/Aqua data are shown in Fig. 4.2a and 4.2b, and their difference $\Delta r_{e, 3.7-2.1}=r_{e, 3.7}-r_{e, 2.1}$ in Fig. 4.2c respectively. Several well-known coastal stratocumulus regions, such as off the coasts of California, Peru, and Namibia/Angola, are clearly seen in the figure. One can also see from Fig. 4.2 that the transition from the coastal stratocumulus cloud regimes to the offshore cumulus cloud regimes is quite sharp. Accompanying this transition, $r_{e, 2.1}$ increases substantially from $8 \sim 10 \mu \mathrm{m}$ near the coast to as large as $20 \sim 25 \mu \mathrm{m}$ far offshore. However, $r_{e, 3.7}$ is significantly smaller than its counterparts. It is easily seen that $\Delta r_{e, 3.7-2.1}$ shows an obvious dependence on cloud regime. For example, over coastal stratocumulus cloud regions, $\Delta r_{e, 3.7-2.1}$ is close to zero, or even slightly positive. However, over the broken cumulus cloud regions, where water cloud fraction is small, $r_{e, 3.7}$ is seen to be smaller than $r_{e, 2.1}$ by as much as $5-10 \mu \mathrm{m}$ on average.

\subsubsection{Correlation with key cloud parameters}

In the rest of this section, we explore the correlations between MODIS $r_{e}$ retrieval differences and several key factors. In doing so, we attempt to identify regimes where the $r_{e}$ retrieval differences can be attributed more to cloud physics, such as drizzle, than to retrieval uncertainties and artifacts caused by, for example, 3D radiative effects.

Figure 4.3a shows the joint histogram between $r_{e, 3.7}$ and $r_{e, 2.1}$ based on about 1.5 billion Level 2 marine water cloud pixels collected during May 2007 by Aqua MODIS from $60 \mathrm{~S}$ to $60 \mathrm{~N}$. Evidently, the density of points is highest along the oneto-one line, attesting that pixel-level $r_{e, 3.7}$ and $r_{e, 2.1}$ modes agree reasonably well. It is interesting to see that the bias between $r_{e, 3.7}$ and $r_{e, 2.1}$ is quite small when $r_{e}$ is smaller than about $12 \sim 13 \mu \mathrm{m}$. However, when $r_{e}$ is larger than about $15 \mu \mathrm{m}$, the histogram distribution is clearly weighted toward the $r_{e, 2.1}$ side and the deviation from the one-to-one line increases with increasing $r_{e, 2.1}$. Figure $4.3 \mathrm{~b}$ presents the same story, but from a different perspective. It shows the joint histogram between $\Delta r_{e, 3.7-2.1}$ and $r_{e, 2.1}$. Note that, in order to reduce the data sampling impact on the shape of the joint histogram, the histogram of $\Delta r_{e, 3.7-2.1}$ at $r_{e, 2.1}$ bin has been normalized with respect to its peak value at that bin. Therefore, the red color in Fig. 4.3b corresponds to the most frequent $\Delta r_{e, 3.7-2.1}$ at a given $r_{e, 2.1}$ bin. The gray dotted line in Fig. 4.3b shows the PDF of $r_{e, 2.1}$ derived from one month of Level 2 data. Interestingly, the bin-normalized histogram of $\Delta r_{e, 3.7-2.1}$ vs. $r_{e, 2.1}$ not only shows an increasing spread in $\Delta r_{e, 3.7-2.1}$ with increasing $r_{e, 2.1}$, but also a clear systematic transition, in terms of the most likely observed $\Delta r_{e, 3.7-2.1}$ for a given $r_{e, 2.1}$ (i.e. the red area), from near-zero values when $r_{e, 2.1}<15 \mu \mathrm{m}$ to larger negative values when $r_{e, 2.1}>15 \mu \mathrm{m}$. The fact that this threshold-like behavior 
(a) $r_{e, 2.1}[\mu \mathrm{m}]$

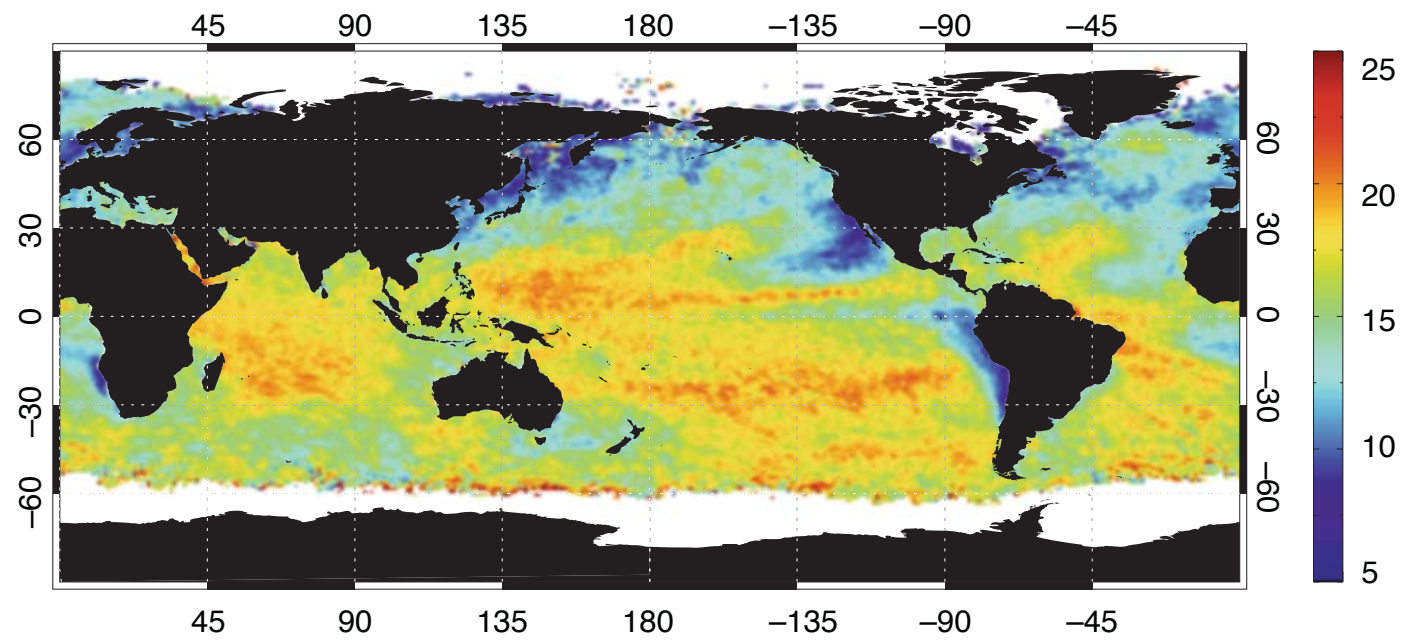

(b) $r_{e, 3.7}[\mu \mathrm{m}]$

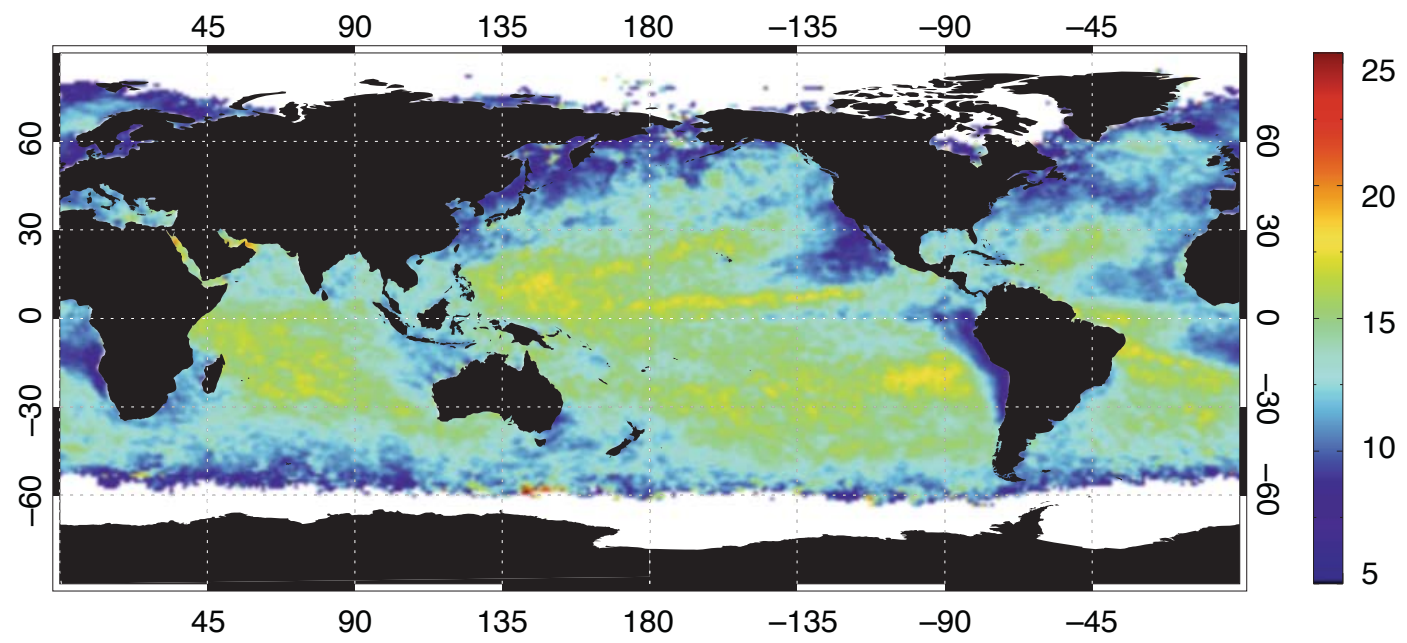

(c) $\Delta r_{e, 3.7-2.1}[\mu \mathrm{m}]$

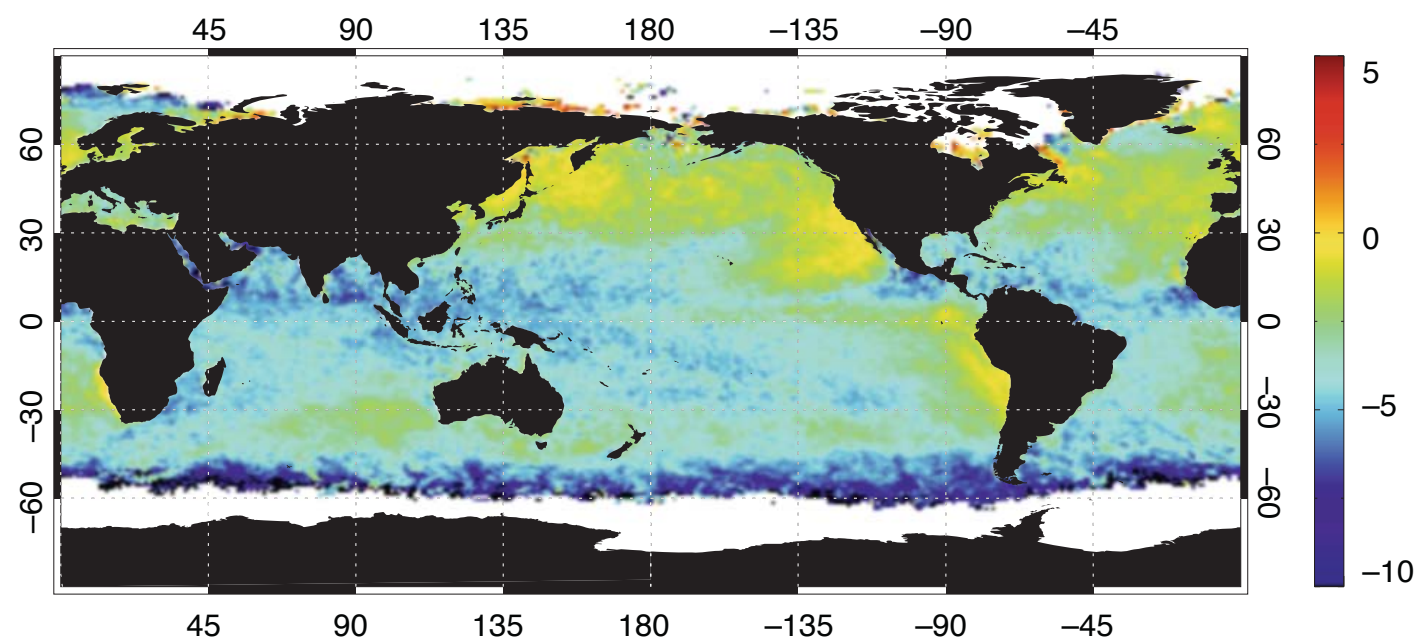

Fig. 4.2. Monthly mean MODIS (a) $r_{e, 2.1}$, (b) $r_{e, 3.7}$, and (c) $\Delta r_{e, 3.7-2.1}$ retrievals for warm (cloud top temperature larger than $273 \mathrm{~K}$ ) liquid-phase clouds over ocean. 
(a)

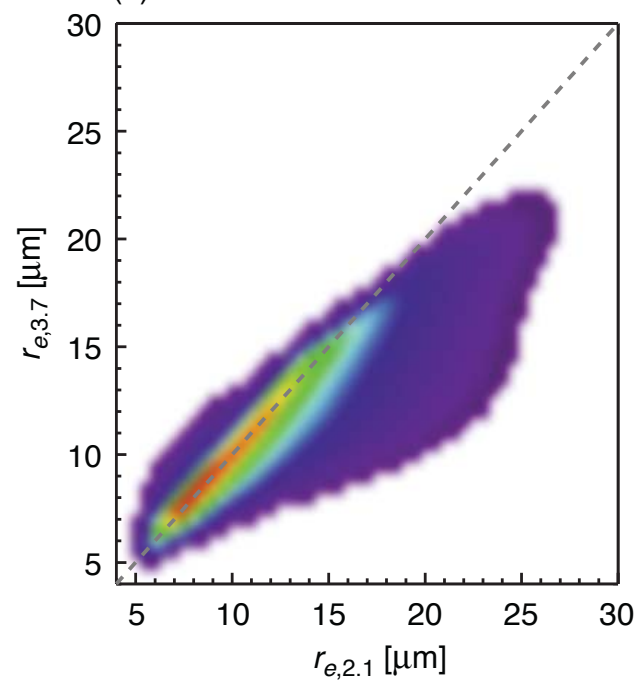

(b)

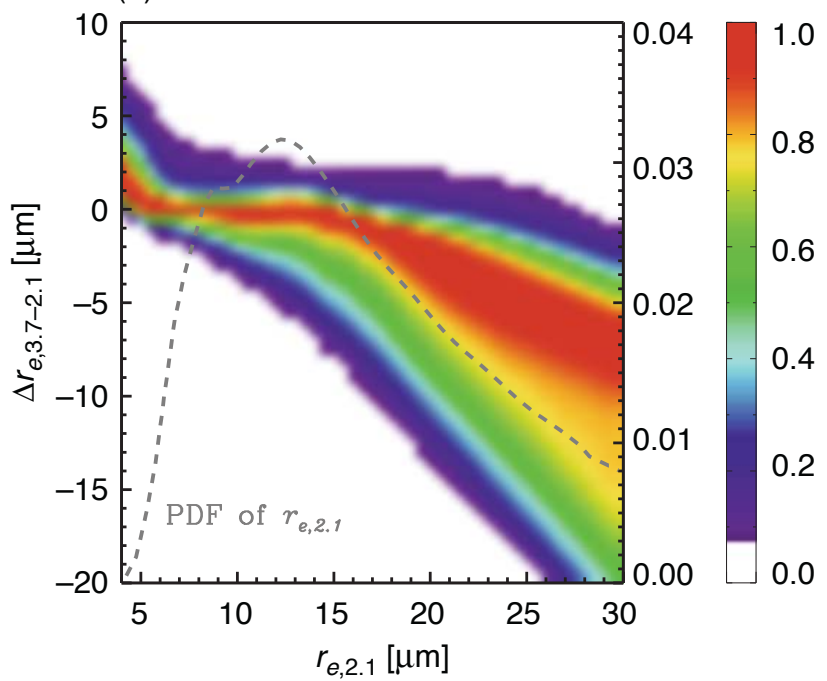

Fig. 4.3. The joint histogram of pixel level (a) $r_{e, 2.1}$, vs. $r_{e, 3.7}$, (b) $\Delta r_{e} 3.7-2.1$ vs. $r_{e, 2.1}$, which is bin-normalized with respect to the PDF of $r_{e, 2.1}$ (gray dashed line).

takes place at $r_{e} \sim 15 \mu \mathrm{m}$ is particularly interesting because $r_{e} \sim 15 \mu \mathrm{m}$ has been suggested to be the threshold for the collision-coalescence process to take place in marine water clouds (Gerber, 1996). However, further studies are needed to determine whether this is simply a coincidence or due to more fundamental physical reasons.

Figure 4.4 shows the bin-normalized joint histograms between $\Delta r_{e, 3.7-2.1}$ and cloud optical thickness $(\tau)$. One can easily note that, when clouds are optically thin (e.g. $\tau<5$ ), $\Delta r_{e, 3.7-2.1}$ varies quite remarkably from $-15 \mu \mathrm{m}$ to $10 \mu \mathrm{m}$. However, when the cloud becomes sufficiently thick $(\tau>5)$, the statistics of $\Delta r_{e, 3.7-2.1}$ become stable and show little dependence on $\tau$. The large variability of $\Delta r_{e, 3.7-2.1}$ for thin clouds in Fig. 4.4 is likely a result of the combined effects of random retrieval uncertainties (see section 4.4.1), 3D radiative effects (section 4.4.5), and the socalled plane-parallel $r_{e}$ retrieval bias (section 4.4.4). For thin clouds, the signal is comparable or smaller than, the noise caused by instrument uncertainties, ancillary data uncertainties, and discretization and interpolation of the LUT. As a result, the uncertainty associated with the MODIS $r_{e}$ retrievals for thin clouds is large. In addition, thin and broken clouds often have significant horizontal heterogeneity, providing favorable conditions for 3D radiative effects and plane-parallel $r_{e}$ retrieval bias. Caution must therefore be taken when interpreting the meaning of $\Delta r_{e, 3.7-2.1}$ for clouds with $\tau<5$ because many factors other than cloud physics, such as retrievals errors and artifacts, all play a significant role in this regime.

When analyzing the potential connection between cloud horizontal inhomogeneity and $r_{e}$ retrieval failure, we will use a so-called sub-pixel inhomogeneity index $\left(H_{\sigma}\right)$ from MODIS. It is defined as (Liang et al., 2009)

$$
H_{\sigma}=\frac{\operatorname{stdev}\left[R_{i}(0.86 \mu \mathrm{m}, 250 \mathrm{~m})\right]}{\operatorname{mean}\left[R_{i}(0.86 \mu \mathrm{m}, 250 \mathrm{~m})\right]},
$$




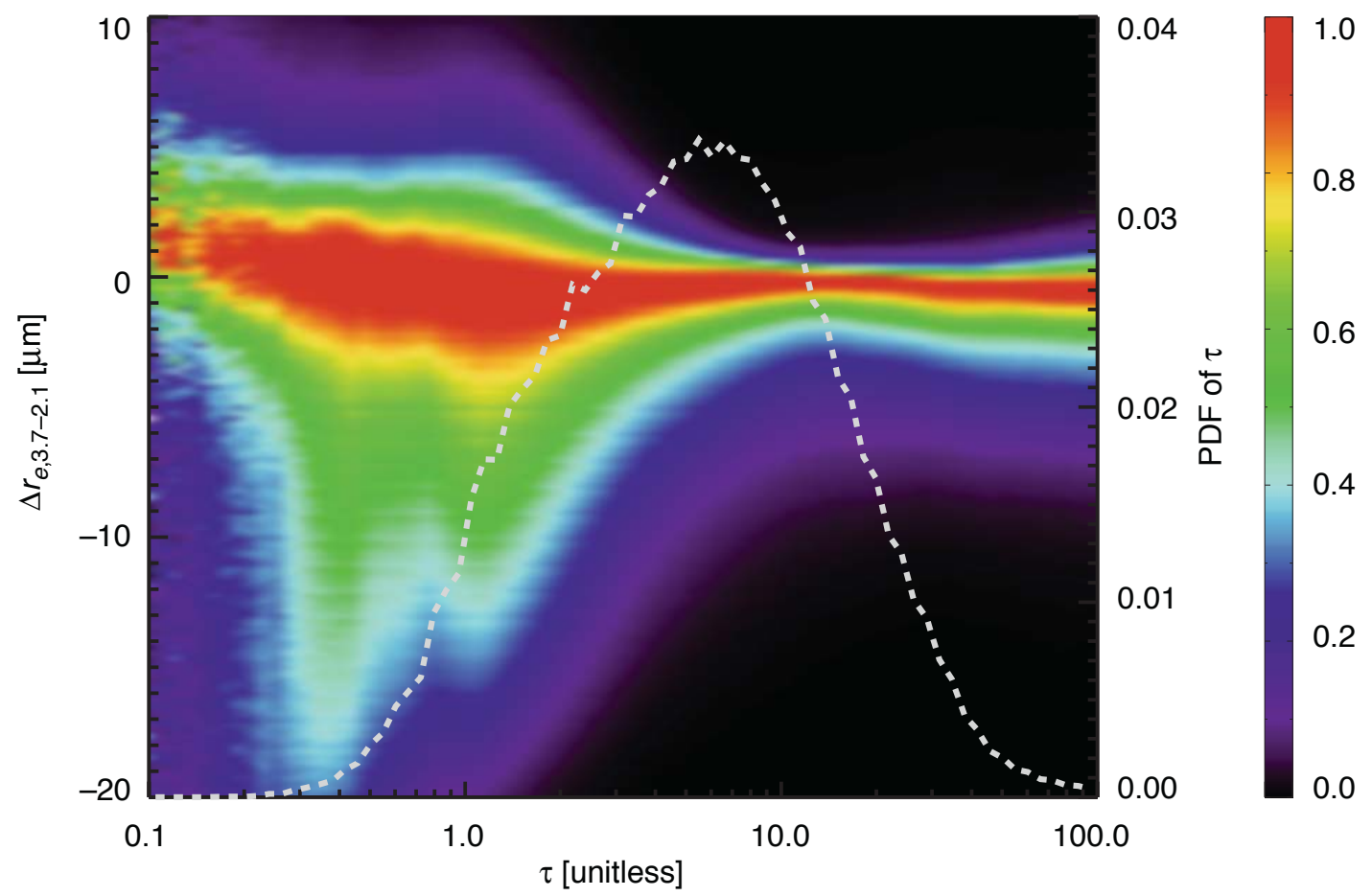

Fig. 4.4. Joint histogram of $\Delta r_{e, 3.7-2.1}$ and cloud optical thickness $(\tau)$, bin-normalized by the PDF of $\tau$.

where $\operatorname{stdev}\left[R_{i}(0.86 \mu \mathrm{m}, 250 \mathrm{~m})\right]$ and $\operatorname{mean}\left[R_{i}(0.86 \mu \mathrm{m}, 250 \mathrm{~m})\right]$ indicate the standard deviation and mean of the measured reflectances, respectively, for the principle 16 250-m-resolution sub-pixels within the 1-km MODIS pixel retrieval footprint. Thus, $H_{\sigma}$ has a spatial resolution (i.e. $1 \mathrm{~km}$ ) consistent with the cloud property retrieval and increases with pixel inhomogeneity. Recent studies found that the $H_{\sigma}$ index derived from high-resolution (250-m) MODIS cloud reflectance measurement provides an objective and quantitative measurement of the horizontal inhomogeneity of 1-km MODIS pixel (Di Girolamo et al., 2010; Zhang and Platnick, 2011; Zhang et al., 2012). The $H_{\sigma}$ index will be reported in the MOD06 product in the coming Collection 6 . The dependence of $\Delta r_{e, 3.7-2.1}$ on cloud horizontal inhomogeneity is shown in Fig. 4.5. Figure 4.5a and 4.5b show the bin-normalized joint histograms of the sub-pixel cloud inhomogeneity $\left(H_{\sigma}\right)$ defined vs. $r_{e, 2.1}$ and $r_{e, 3.7}$, respectively. Figure $4.5 \mathrm{c}$ shows the bin-normalized joint histograms of the $H_{\sigma}$ vs. $\Delta r_{e, 3.7-2.1}$. Optically thin clouds $(\tau<5)$ are excluded from this figure for the above-mentioned reason, but results are similar if we include thin pixels (not shown). The most compelling feature in Fig. 4.5a is the sharp transition of $r_{e, 2.1}$ at $H_{\sigma}$ around $0.3 \sim 0.5$. When $H_{\sigma}$ is smaller than 0.3 , the most likely $r_{e, 2.1}$ (i.e. red area in Fig. 4.5a) for a given $H_{\sigma}$ stays relatively constant, within $10 \sim 15 \mu \mathrm{m}$. However, when $H_{\sigma}$ exceeds about 0.3 , the most likely $r_{e, 2.1}$ value increases dramatically with $H_{\sigma}$. Interestingly, this is not the case in Fig. 4.5b, where the most likely value of $r_{e, 3.7}$ shows only weak dependence on the sub-pixel inhomogeneity. It is therefore not surprising to see in Fig. $4.5 \mathrm{c}$ the most likely values of $\Delta r_{e, 3.7-2.1}$ 

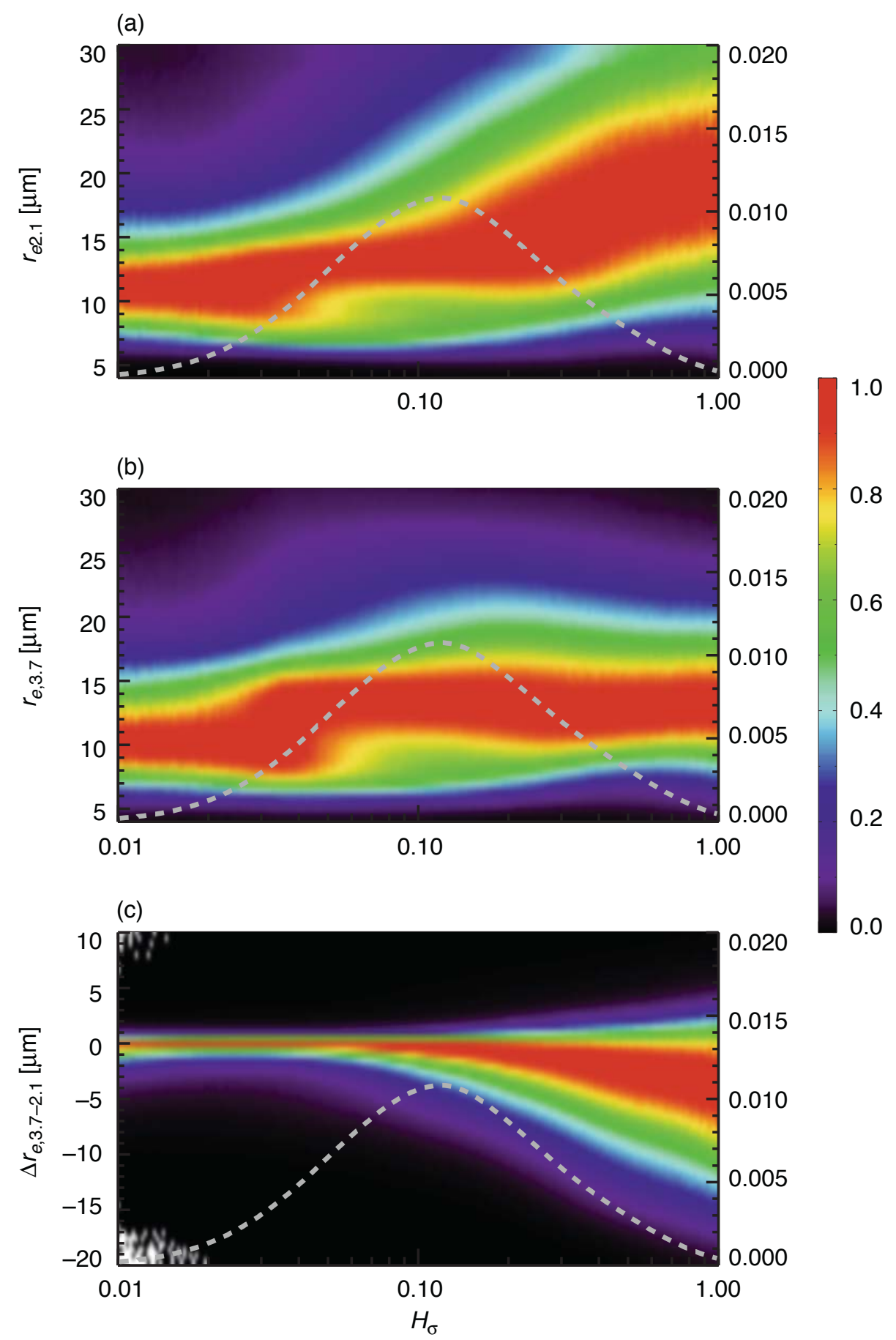

Fig. 4.5. Joint histograms of (a) $r_{e, 2.1}$ vs. sub-pixel inhomogeneity index $\left(H_{\sigma}\right)$, (b) $r_{e, 3.7}$ vs. $H_{\sigma}$, and (c) $\Delta r_{e, 3.7-2.1}$ vs. $H_{\sigma}$. All histograms in this figure are bin-normalized with respect to the PDF of $H_{\sigma}$. Note that thin clouds with $\tau<5$ have been excluded in this figure, but results are similar if we include thin pixels (not shown).

shifting from near-zero to the negative side when $H_{\sigma}$ exceeds about 0.3 . The potential reasons for the dependence of $\Delta r_{e, 3.7-2.1}$ on cloud inhomogeneity $H_{\sigma}$ will be discussed later in sections 4.4.4 and 4.4.5. 
In addition to $r_{e}, \tau$, and $H_{\sigma}$, we have also investigated the dependence of $\Delta r_{e, 3.7-2.1}$ on other factors such as cloud top temperature, solar zenith angle, satellite viewing angle, scattering angle, surface reflectance, etc., none of which shows an impact on $\Delta r_{e, 3.7-2.1}$ as dramatic and clear as $r_{e}, \tau$, and $H_{\sigma}$.

\subsection{Potential reasons for the spectral difference}

The fact that $\Delta r_{e, 3.7-2.1}$ is correlated with several key cloud parameters, including $r_{e}, \tau$, and $H_{\sigma}$, indicates that the spectral dependence of the MODIS $r_{e}$ retrieval is a complex issue likely caused by multiple mechanisms. Indeed, several hypotheses have been proposed to explain the causes of $\Delta r_{e, 3.7-2.1}$ and its behaviors described in the previous section. This section provides an overview of these hypotheses. It is helpful to begin with a classification. The existing hypotheses can be grouped into two categories based on their underlying mechanisms and consequent implications. In one category are those related to the inherent limitations of the bi-spectral method, such as retrieval uncertainties (section 4.4.1), the sub-pixel inhomogeneity (section 4.4.4), and lack of consideration of 3D radiative effects (section 4.4.5) in the retrieval method. In such cases, $\Delta r_{e, 3.7-2.1}$ carries little, if any, information about the microphysical property of the pixel, but is rather a retrieval artifact indicating significant uncertainties in either $r_{e, 2.1}$ or $r_{e, 3.7}$, or both. In the other category are those related to the fact that $r_{e, 3.7}$ and $r_{e, 2.1}$ have different sensitivities to cloud vertical structure (section 4.4.2) and the presence of large drizzle drops (section 4.4.3) in warm water clouds. The implication of this is that the spectral $r_{e}$ difference actually contains useful information about the cloud that can be used and actually has been used for remote sensing and model validation.

\subsubsection{Random error}

As shown in Fig. 4.4, $\Delta r_{e, 3.7-2.1}$ for clouds with $\tau<5$ varies more widely than that for thicker clouds. The large variation of $\Delta r_{e, 3.7-2.1}$ for thin clouds is likely caused by random retrieval uncertainties. There are many sources of uncertainty in the MODIS retrieval, such as instrument uncertainties, ancillary data uncertainties, and discretization and interpolation of the LUT. These sources are usually uncorrelated, leading to random errors in the observed cloud reflectances. When the cloud is thin, the signal from the cloud is comparable to, or even smaller than, the retrieval uncertainties, which can result in large errors in $r_{e, 2.1}$ and $r_{e, 3.7}$, and a highly variable $\Delta r_{e, 3.7-2.1}$. An example to illustrate the impact of random uncertainties on MODIS $\tau$ and $r_{e}$ retrievals is given in Fig. 4.6. In the example, one point in the MODIS operational LUT, with $\tau=4.1$ and $r_{e}=16 \mu \mathrm{m}$, (i.e. the center of the cross in Fig. 4.1), is chosen for the purpose of illustration. Uncorrelated random errors, which are assumed to follow the normal distribution with standard deviation $\sigma=10 \%$, are added to the reflectances of this point at all three bands to mimic the above-mentioned retrieval uncertainties. The magnitude (one standard deviation) of error is indicated by the size of the crosses in Fig. 4.1. One million such samples are generated and then used to obtained $\tau$ and $r_{e}$ retrieval samples. The probability density functions $(\mathrm{PDF})$ of $\tau$ retrievals are shown in Fig. 4.6a. 
The two $\tau$ retrievals, one based on $0.86-\mu \mathrm{m}$ and $2.1-\mu \mathrm{m}$ band LUT and the other based $0.86-\mu \mathrm{m}$ and $3.7-\mu \mathrm{m}$ band LUT, both vary closely around the true value of $\tau=4.1$ and they agree with each other very well. The PDFs of $r_{e, 2.1}, r_{e, 3.7}$, and $\Delta r_{e, 3.7-2.1}$ are shown in Fig. 4.6b. Interestingly, $r_{e, 2.1}$ has a narrower PDF than $r_{e, 3.7}$, even though the same magnitude of error (i.e. $10 \%$ in reflectance) is given to the two bands. This difference is mainly due to the fact that the 0.86$\mu \mathrm{m}$ and $2.1-\mu \mathrm{m}$ band LUT ( $L U T_{0.86 \& 2.1}$ hereafter) is generally more condensed in the $r_{e}$ direction than the $0.86-\mu \mathrm{m}$ and $3.7-\mu \mathrm{m}$ band LUT ( $L U T_{0.86 \& 3.7}$ ) (i.e. $\left.\left|\partial \ln R(2.1 \mu \mathrm{m}) / \partial r_{e}\right|<\left|\partial \ln R(3.7 \mu \mathrm{m}) / \partial r_{e}\right|\right)$. As a result, the same magnitude of reflectance error (e.g. 10\%) leads to different $r_{e}$ error. The fact that $L U T_{0.86 \& 2.1}$ is generally less orthogonal than $L U T_{0.86 \& 3.7}$ also plays a role. The orthogonality of LUT is determined by the extent to which the cloud reflectance in the SWIR band is independent from that in the $0.86-\mu \mathrm{m}$ band. Because is generally more orthogonal than (i.e. $|\partial R(2.1 \mu \mathrm{m}) / \partial R(0.86 \mu \mathrm{m})|>|\partial R(3.7 \mu \mathrm{m}) / \partial R(0.86 \mu \mathrm{m})|)$, error in the $0.86-\mu \mathrm{m}$ band has less impact on $r_{e, 3.7}$ than $r_{e, 2.1}$, which contributes to the PDF difference between $r_{e, 2.1}$ and $r_{e, 3.7}$ in Fig. 4.6b. This effect of LUT orthogonality is demonstrated in Fig. 4.6c and 4.6d, which shows the PDFs of $\tau$ retrievals (Fig. 4.6c), $r_{e, 2.1}, r_{e, 3.7}$, and $\Delta r_{e, 3.7-2.1}$ (Fig. 4.6d) when we only add random errors to $R(0.86 \mu \mathrm{m})$ but not to $R(2.1 \mu \mathrm{m})$ and $R(3.7 \mu \mathrm{m})$. Due to the LUT orthogonality difference, the error, which is solely in the $R(0.86 \mu \mathrm{m})$, causes only a small error in $r_{e, 3.7}$ but causes a significant error in $r_{e, 2.1}$ retrievals. This difference in error between the two bands leads to $\Delta r_{e, 3.7-2.1}$ varying between $-5 \mu \mathrm{m}$ and $5 \mu \mathrm{m}$. It is worth mentioning here that the orthogonality of LUT also plays an important role in the so-called plane-parallel bias that will be discussed in section 4.4.4. As a result, the widths of the $r_{e, 2.1}, r_{e, 3.7}$, and the $\Delta r_{e, 3.7-2.1}$ PDFs in Fig. $4.6 \mathrm{~b}$ are quite wide, which suggests that retrieval uncertainties caused by instrument uncertainties, ancillary data uncertainties, etc. probably play an important role in causing the large variation of $\Delta r_{e, 3.7-2.1}$ for thin clouds in Fig. 4.4.

As shown in Fig. 4.6, random error in reflectance tends to result in random error in $r_{e, 2.1}$ and $r_{e, 3.7}$, which in turn leads to a $\Delta r_{e, 3.7-2.1}$ with a mean value close to zero. Therefore, the random retrieval uncertainties cannot explain why $r_{e, 3.7}$ is systematically smaller than $r_{e, 2.1}$, especially when cloud is thick (i.e. high signal-to-noise ratio).

\subsubsection{Vertical cloud structure}

Several studies have shown that $r_{e, 2.1}$ and $r_{e, 3.7}$ retrieval have different sensitivities to the in-cloud vertical structure of cloud droplet microphysics, leading to spectral difference in $r_{e}$ retrieval (Platnick, 2000; Kokhanovsky, 2004; Nakajima et al., 2010a; Zhang et al., 2010). The concept of 'weighting function' developed by Platnick (2000) provides a convenient framework to assess the sensitivity of $r_{e}$ retrieval based on the bi-spectral method to the vertical structure of cloud. One of the two weighting functions introduced in Platnick (2000) is based on the maximum penetration depth of photon into cloud. This weighting function, $w_{m}\left(\tau, \tau_{c}\right)$, is defined as

$$
w_{m}\left(\tau, \tau_{c}\right)=\frac{1}{R\left(\tau_{c}\right)} \frac{d R(\tau)}{d \tau}
$$



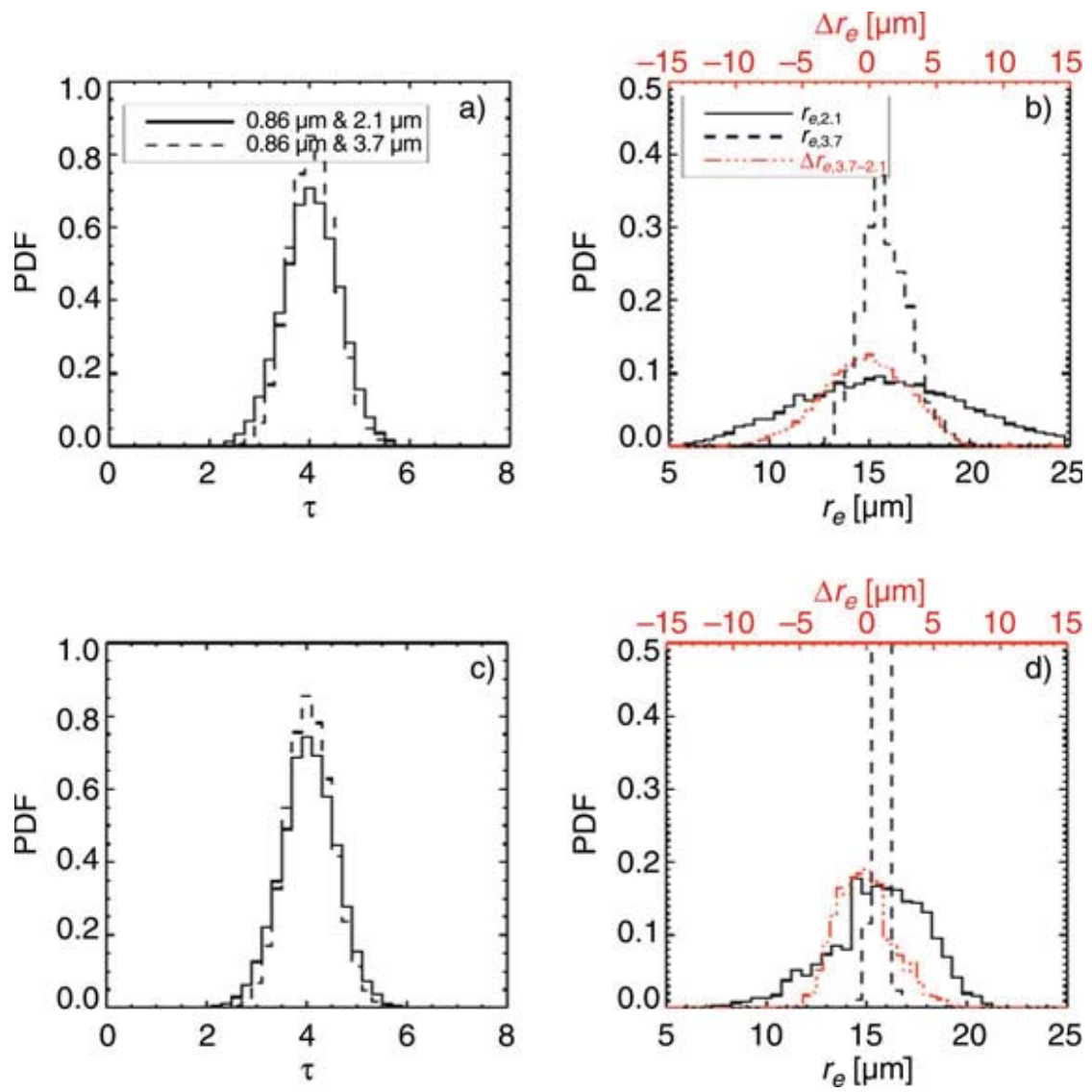

Fig. 4.6. An example to illustrate the impact of random error in cloud reflectance on $\tau$ and $r_{e}$ retrievals. In (a) and (b), random error is assigned to both the $0.86-\mu \mathrm{m}$ band and the SWIR band (2.1- $\mu \mathrm{m}$ or 3.7- $\mu \mathrm{m}$ band). In (c) and (d), random error is only assigned to the SWIR band. Impacts on $\tau$ retrievals are shown in (a) and (c). Impacts on $r_{e}$ retrievals and $\Delta r_{e}$ are shown in (b) and (d). See text for details.

where $\tau_{c}$ is the total cloud optical thickness, $\tau$ is the optical depth of a level in cloud from cloud top, $R\left(\tau_{c}\right)$ is the cloud reflectance, and $d R(\tau)=R(\tau+d \tau)-R(\tau)$ represents the fraction of all reflected photons that penetrate to a maximum optical depth between $\tau$ and $\tau+d \tau$. As shown in Platnick (2000), the shape of $w_{m}\left(\tau, \tau_{c}\right)$ can be used to quantitatively interpret the impact of the in-cloud vertical structure on MODIS retrievals. Additionally, it is shown that the value predicted from the equation

$$
r_{e}^{*}=\int_{0}^{\tau_{c}} r_{e}(\tau) w_{m}\left(\tau, \tau_{c}\right) d \tau
$$

where $r_{e}^{*}$, is the expected $r_{e}$ retrieval value based on the vertical weighting, agrees to within $\pm 1 \mu \mathrm{m}$ with the value from full radiative transfer simulation. Note that the vertical structure of cloud has little impact on cloud optical thickness retrieval.

Figure 4.7 shows an example of $w_{m}\left(\tau, \tau_{c}\right)$ for $r_{e 2.1}$ (black line) and $r_{e 3.7}$ (red line) for a cloud layer with adiabatic cloud vertical structure, as shown by the dashed $r_{e}(\tau)$ line. The $w_{m}\left(\tau, \tau_{c}\right)$ for $r_{e 3.7}$ peaks at cloud top, while $w_{m}\left(\tau, \tau_{c}\right)$ for $r_{e 2.1}$ peaks lower in the cloud. This difference and Eq. (4.5) suggest that $r_{e 3.7}$ retrieval 


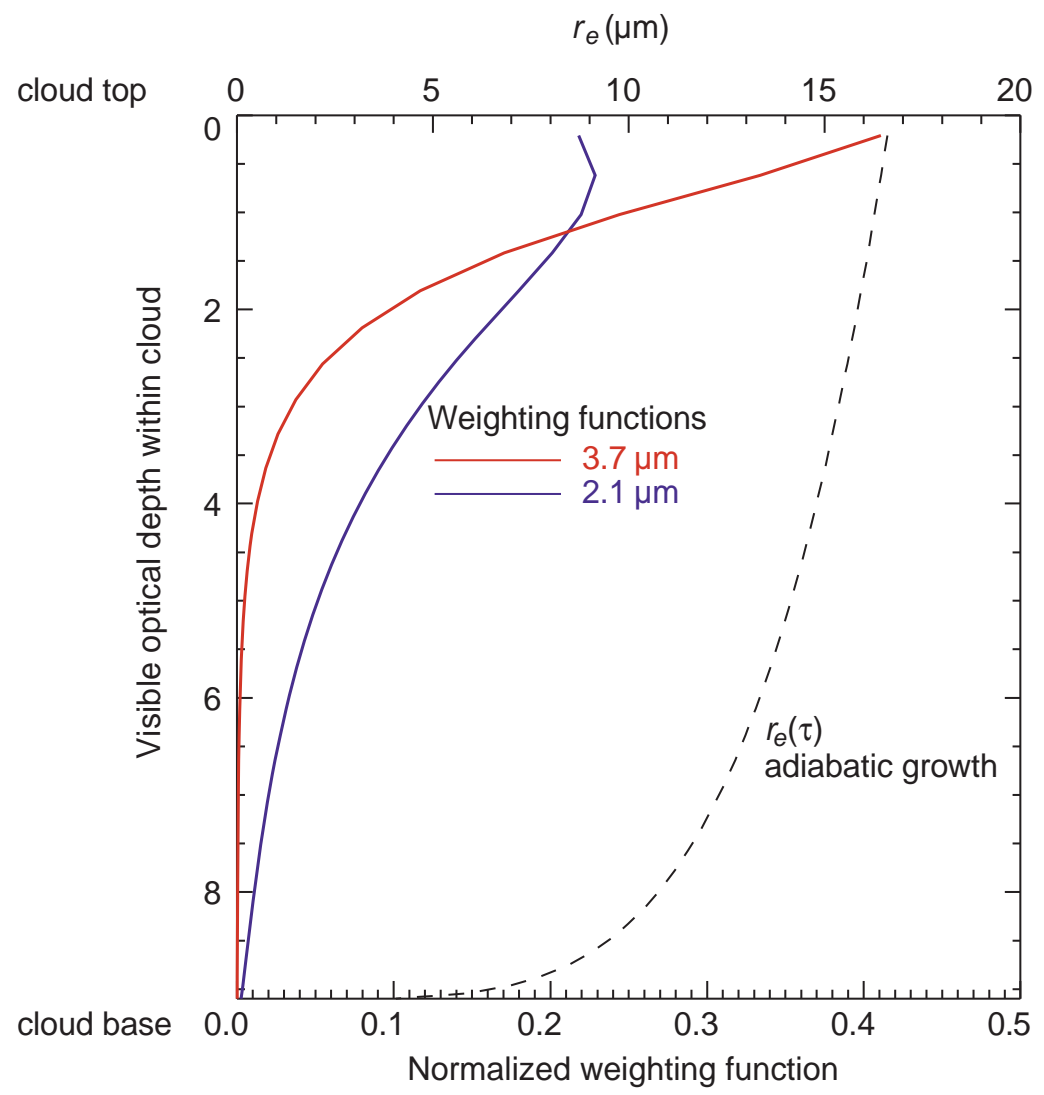

Fig. 4.7. Normalized weighting function $w_{m}\left(\tau, \tau_{c}\right)$ for $r_{e 2.1}$ (blue line) and $r_{e 3.7}$ (red line) as a function of cloud optical depth for a cloud with adiabatic vertical structure. Dashed line shows the vertical variation of $r_{e}$ as a function of cloud optical depth.

has more sensitivity to the cloud microphysics at the uppermost part of cloud while $r_{e 2.1}$ retrieval penetrates deeper into the cloud with maximum sensitivity at about $\tau \sim 2$ or 3. For the case in Fig. 4.7, $r_{e 2.1}$ and $r_{e 3.7}$ predicted from $w_{m}\left(\tau, \tau_{c}\right)$ based on Eq. (4.5) are $15.2 \mu \mathrm{m}$ and $16.1 \mu \mathrm{m}$. Both agree well with full retrievals.

It is important to note that the general shape of the weighting function is not sensitive to the detailed cloud vertical structure. Zhang et al. (2010) demonstrated that the weighting functions for two cases, one with $r_{e}(\tau)$ increasing and the other decreasing from cloud top toward cloud base, are quite similar to each other. The invariance of the weighting function shape, according to Eq. (4.5), suggests that the retrieved $r_{e}^{*}$ is largely determined by cloud vertical structure $r_{e}(\tau)$. And so is $\Delta r_{e, 3.7-2.1}$ as far as cloud vertical structure is concerned. As a consequence, $r_{e, 3.7}>r_{e, 2.1}\left(\Delta r_{e, 3.7-2.1}>0\right)$ according to Eq. (4.5) when the cloud droplet size increases from cloud base toward cloud top, and vice versa when cloud droplet size decreases from cloud base toward cloud top. Various microphysical processes can affect cloud vertical structure, such as adiabatic growth, entrainment mixing, collision-coalescence, and sedimentation. It is therefore important to understand the influence of these processes on the vertical structure of cloud microphysics and their consequent impact on $\Delta r_{e, 3.7-2.1}$.

In a classic adiabatic growth model (Brenguier et al., 2000), cloud $r_{e}$ increases monotonically from cloud base to cloud top. In such a case, $r_{e, 3.7}$ should be larger 
than $r_{e, 2.1}$, as shown in the example in Fig. 4.7. Indeed, some recent studies, such as Painemal and Zuidema (2011) and Zhang and Platnick (2011), have found some positive $\Delta r_{e, 3.7-2.1}$, which is qualitatively consistent with the classic adiabatic growth model as shown in Fig. 4.7. However, as mentioned in section 4.3, only a small fraction of MODIS MBL cloud observations have positive $\Delta r_{e, 3.7-2.1}$. Moreover, because the adiabatic condensation growth becomes less efficient as the droplet grows larger, the droplet growth rate $d r_{e} / d z$ remains relative small over the upper portion of the water cloud (Brenguier et al., 2000). As a result, the magnitude of $\Delta r_{e, 3.7-2.1}$ induced by adiabatic cloud vertical structure is expected to be small, generally smaller than $2 \mu \mathrm{m}$ (Platnick, 2000).

Cloud top entrainment could lead to a decreasing-with-height $r_{e}$ structure at cloud top. Several recent studies speculated that this cloud top entrainmentinduced structure plays an important role in causing smaller $r_{e, 3.7}$ retrieval than $r_{e, 2.1}$ (Breon and Doutriaux-Boucher, 2005; Seethala and Horváth, 2010). It should be pointed out that the decreasing-with-height $r_{e}$ structure at cloud top assumed in these studies seems to indicate a homogenous mixing process (Baker et al., 1980). On the contrary, observational studies have actually found more inhomogeneous mixing cases than homogenous mixing cases (Gerber et al., 2005). A couple of recent studies analyzed in situ measurements of MBL clouds off the coast of Peru made during the VOCALS-REx (or American Monsoon Systems (VAMOS) Ocean-Cloud-Atmosphere-Land Study Regional Experiment) campaign (Painemal and Zuidema, 2011; King et al., 2012). They did not observe the decreasing-withheight $r_{e}$ structure at cloud top. In addition, the strong correlation of $\Delta r_{e, 3.7-2.1}$ with $r_{e, 2.1}$ and the threshold behavior of $\Delta r_{e, 3.7-2.1}$ at $r_{e, 2.1} \sim 15 \mu \mathrm{m}$ (Fig. 4.3) do not easily fit into the cloud top entrainment structure argument.

Warm rain processes (e.g. collision-coalesce) give rise to embryo drizzle drops at cloud top, and also make them grow bigger as they fall from the cloud top, potentially leading to a decreasing-with-height $r_{e}$ structure from cloud top toward cloud base (Berry, 1967; Berry and Reinhardt, 1974; Pruppacher and Klett, 1997). Therefore, cloud vertical structure induced by warm rain processes has been argued in many studies to be the primary reason for the large negative $\Delta r_{e, 3.7-2.1}$ (Chang and Li, 2002, 2003; Chen et al., 2007; Nakajima et al., 2010a, 2010b; Kokhanovsky and Rozanov, 2011). Some behaviors of $\Delta r_{e, 3.7-2.1}$ shown in section 4.3 seem in favor of this argument. For example, it is seen in Fig. $4.3 \mathrm{~b}$ that $\Delta r_{e, 3.7-2.1}$ is close to zero when $r_{e, 2.1}<15 \mu \mathrm{m}$ and decreases quickly with $r_{e, 2.1}$ when $r_{e, 2.1}>$ $15 \mu \mathrm{m}$. This threshold behavior could be explained as a result of increasing drizzle probability when cloud $r_{e}$ becomes larger than $15 \mu \mathrm{m}$ (Gerber, 1996; Nakajima et al., 2010a, 2010b). Although there is increasing evidence indicating that warm rain processes play an important role in causing the large negative $\Delta r_{e, 3.7-2.1}$, there are also studies suggesting otherwise. For example, a couple of case studies based on large-eddy simulation of MBL clouds and radiative transfer simulations found only a negligible impact of drizzle on both $r_{e}$ retrievals and thereby $\Delta r_{e, 3.7-2.1}$ (Zinner et al., 2010; Zhang et al., 2012). Although it is difficult to draw any statistical conclusions from these case studies, they demonstrated a new path toward better understanding the impact of drizzle on MODIS $r_{e}$ retrieval that is worthy of further exploration. 
Finally, it should be noted that some $\Delta r_{e, 3.7-2.1}$ behaviors, the correlation of $\Delta r_{e, 3.7-2.1}$ with cloud horizontal inhomogeneity index $H_{\sigma}$ shown in section 4.3 in particular, cannot be easily explained by cloud vertical structure. This indicates that other factors also have a role.

\subsubsection{Cloud droplet size distribution}

As mentioned in section 4.2, in the operational MODIS retrieval algorithm, cloud DSD follows the monomodal Gamma distribution with fixed effective variance $v_{e}=0.1$ (i.e. Eq. (4.2)). Several studies have shown that, when cloud microphysics deviates from what is assumed in the MODIS retrieval algorithm, the retrieved $r_{e}$ also deviates from the true $r_{e}$. Invalid microphysics assumptions affect $r_{e, 2.1}$ and $r_{e, 3.7}$ retrievals to a different extent, leading to spectral differences (Chang and Li, 2001; Minnis et al., 2004; Zhang, 2013).

The monomodal Gamma distribution assumption for cloud DSD is especially problematic when MBL clouds are precipitating, because warm rain processes (e.g. collision-coalescence) could broaden cloud DSD and even give rise to a second mode, the so-called drizzle or rain mode, creating bi-modal DSD (Berry, 1967; Berry and Reinhardt, 1974; Pruppacher and Klett, 1997). In such a case, the MODIS retrieval process is to find an $r_{e}^{*}$ (the superscript * is to indicate that it is a retrieved value under monomodal DSD assumption) that satisfies the following equation:

$$
\left.R_{\lambda, L U T}\left(r_{e}^{*}\right)\right|_{v_{e}}=R_{\lambda}[n(r)],
$$

where $R_{\lambda}$ is the observed SWIR band cloud reflectance that is a function of the true bi-modal cloud DSD $n(r)$ and $R_{\lambda, L U T}$ is the SWIR band cloud reflectance in the LUT which is pre-computed based on the monomodal DSD assumption. Additionally, $\lambda$ denotes the wavelength of the SWIR band used for $r_{e}$ retrieval (e.g. $2.1 \mu \mathrm{m}$ or $3.7 \mu \mathrm{m})$.

Recently, Zhang (2013) developed a semi-analytical model to explain and predict MODIS $r_{e}$ retrieval results from Eq. (4.6). In a numerical test, the $r_{e}$ retrievals predicted by this model agree well with numerical solutions based on radiative transfer simulations (Zhang, 2013). The model is based on the fact that dependence of $R_{\lambda}$ on cloud microphysics arises mainly from the dependence of cloud single-scattering albedo $\omega_{\lambda}$ on cloud microphysics (Zhang et al., 2009). Figure 4.8a shows the $\omega_{2.1 \mu \mathrm{m}}$ (solid blue line) and $\omega_{3.7 \mu \mathrm{m}}$ (solid red line) of individual cloud droplets computed using a MIE code (Wiscombe, 1979) as a function of droplet radius over the range $r \in[1 \mu \mathrm{m}, 1000 \mu \mathrm{m}]$. Figure 4.8b shows the so-called bulk scattering albedo $\bar{\omega}_{2.1 \mu \mathrm{m}, L U T}$ and $\bar{\omega}_{3.7 \mu \mathrm{m}, L U T}$ in the MODIS LUT, which are averaged over monomodal Gamma DSD, for $r_{e} \in[5 \mu \mathrm{m}, 30 \mu \mathrm{m}]$. Zhang (2013) expanded $\omega_{2.1 \mu \mathrm{m}}$ and $\omega_{3.7} \mu \mathrm{m}$ into polynomials of $r$ :

$$
\omega_{\lambda}(r)=\sum_{i=0}^{N_{\lambda}} k_{i, \lambda} r^{i},
$$

where $k_{i, \lambda}$ are fitting constants given in Table 4.1 , and $\bar{\omega}_{2.1 \mu \mathrm{m}, L U T}$ and $\bar{\omega}_{3.7 \mu \mathrm{m}, L U T}$ into a linear function of $r_{e}$ :

$$
\bar{\omega}_{\lambda}\left(r_{e}\right) \approx \bar{k}_{0, \lambda}+\bar{k}_{1, \lambda} r_{e}
$$


Table 4.1. Fitting coefficients in Eq. (4.7) and the coefficients (values in parentheses) for each nonlinear term in Eq. (4.9). The unit of droplet radius in Eq. (4.7) is micron.

\begin{tabular}{lllllll}
\hline & $\begin{array}{l}k_{0} \\
\left(r_{0}\right)\end{array}$ & $\begin{array}{l}k_{1} \\
\left(k_{1} / \bar{k}_{1}\right)\end{array}$ & $\begin{array}{l}k_{2} \\
\left(k_{2} / \bar{k}_{1}\right)\end{array}$ & $\begin{array}{l}k_{3} \\
\left(k_{3} / \bar{k}_{1}\right)\end{array}$ & $\begin{array}{l}k_{4} \\
\left(k_{4} / \bar{k}_{1}\right)\end{array}$ & $\begin{array}{l}k_{5} \\
\left(k_{5} / \bar{k}_{1}\right)\end{array}$ \\
\hline $2.1 \mu \mathrm{m}$ & $9.952 \mathrm{E}-01$ & $-1.736 \mathrm{E}-03$ & $2.442 \mathrm{E}-06$ & $-1.187 \mathrm{E}-09$ & & \\
& $(1.17 \mu \mathrm{m})$ & $(9.266 \mathrm{E}-01)$ & $(-1.303 \mathrm{E}-03)$ & $(6.335 \mathrm{E}-07)$ & & \\
$3.7 \mu \mathrm{m}$ & $9.623 \mathrm{E}-01$ & $-6.443 \mathrm{E}-03$ & $3.348 \mathrm{E}-05$ & $-7.606 \mathrm{E}-08$ & $7.757 \mathrm{E}-11$ & $-2.907 \mathrm{E}-14$ \\
& $(1.74 \mu \mathrm{m})$ & $(9.314 \mathrm{E}-01)$ & $(-4.840 \mathrm{E}-03)$ & $(1.099 \mathrm{E}-05)$ & $(-1.121 \mathrm{E}-08)$ & $(4.201 \mathrm{E}-12)$ \\
\hline
\end{tabular}

where fitting constants $\bar{k}_{0, \lambda}$ and $\bar{k}_{1, \lambda}$ are given in Table 4.2 . Without going into detail, Zhang (2013) arrived at the following equation to explain and predict MODIS $r_{e}$ retrieval results from Eq. (4.6):

$$
r_{e, \lambda}^{*}=r_{0, \lambda}+\frac{k_{1, \lambda}}{\bar{k}_{1, \lambda}} r_{e}+\sum_{i=2}^{N_{\lambda}} \frac{k_{i, \lambda}}{\bar{k}_{1, \lambda}} \frac{\left\langle r^{i+2}\right\rangle}{\left\langle r^{2}\right\rangle}
$$

where $r_{e, \lambda}^{*}$ is the predicted MODIS $r_{e}$ retrieval, $\left\langle r^{i}\right\rangle=\int_{0}^{\infty} r^{i} n(r) d r$ is the $i$ th moment of the true DSD, and $r_{0, \lambda}=\left(k_{0, \lambda}-\bar{k}_{0, \lambda}\right) / \bar{k}_{\lambda}$ is a constant. With the coefficients known, $r_{e}^{*}$ can be easily solved from the above equation once the $\left\langle r^{i+2}\right\rangle /\left\langle r^{2}\right\rangle$ terms are derived from any given DSD.

Table 4.2. Fitting coefficients in Eq. (4.8). The unit of droplet radius in Eq. (4.8) is micron.

\begin{tabular}{lll}
\hline & $\bar{k}_{0}$ & $\bar{k}_{1}$ \\
\hline $2.1 \mu \mathrm{m}$ & $9.974 \mathrm{E}-01$ & $-1.874 \mathrm{E}-03$ \\
$3.7 \mu \mathrm{m}$ & $9.744 \mathrm{E}-01$ & $-6.918 \mathrm{E}-03$ \\
\hline
\end{tabular}

It can be seen from Table 4.1 that $k_{1, \lambda} / \bar{k}_{\lambda}$ is close to unity, which suggests that $r_{e}^{*}$ should be close to the true value of $r_{e}$, if the higher-order terms, namely $\left(k_{i, \lambda}\left\langle r^{i+2}\right\rangle\right) /\left(\bar{k}_{\lambda}\left\langle r^{2}\right\rangle\right)$, in Eq. (4.9) are small. The magnitude of higher-order terms is determined by two competing factors. On one hand, the $\left\langle r^{i+2}\right\rangle /\left\langle r^{2}\right\rangle$ term, which is in the same order of magnitude as $r_{e}^{i}$, increases algorithmically with $i$. On the other hand, as seen in Table 4.1 , the $k_{i, \lambda} / \bar{k}_{1, \lambda}$ term decreases with polynomial order $i$. Provided that $r_{e}$ ranges generally from a few to a few tens of microns, the decrease of $k_{i, \lambda} / \bar{k}_{1, \lambda}$ terms can be expected to be dominant, leading to a decreasing impact of nonlinear terms on $r_{e}^{*}$ with increasing order $i$. Therefore, the most important nonlinear term is the second-order term $\left(i=2\right.$ ). Because $k_{2, \lambda} / \bar{k}_{1, \lambda}<0$ (see Table 4.1) and $\left\langle r^{4}\right\rangle /\left\langle r^{2}\right\rangle>0$, the sign of this term is negative, implying that $r_{e}^{*}$ tends to underestimate the true value when this term is significant. Physically, this term is a result of the nonlinear relationship between $\bar{\omega}_{\lambda}$ and $r$. As seen in Fig. 4.8a, $\omega_{\lambda}$ decreases monotonically with $r$, and the rate becomes slower as $\omega_{\lambda}$ approaches 
a) Single-scattering properties vs. $r$

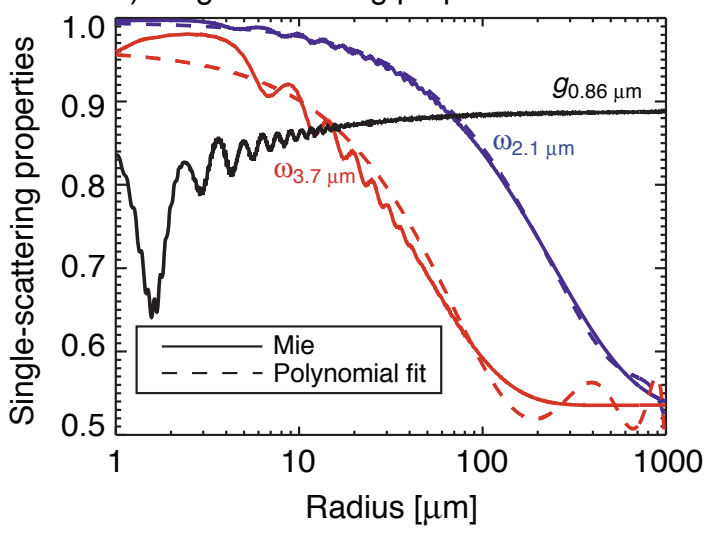

b) Bulk scattering properties vs. $r_{e}$

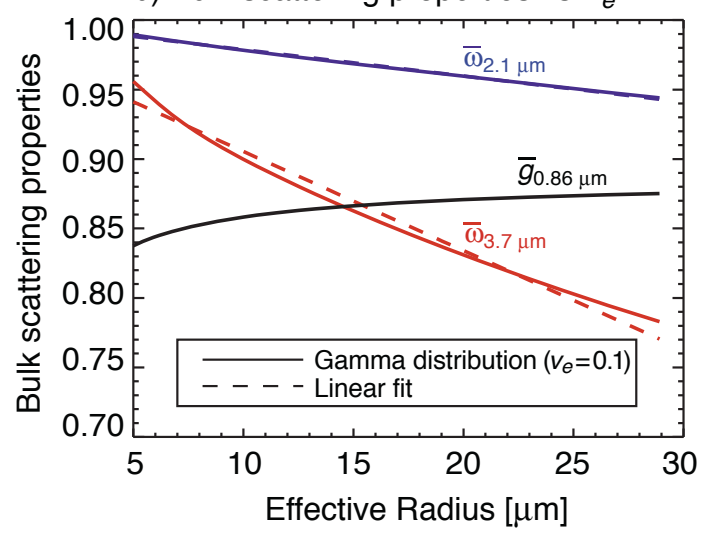

Fig. 4.8. (a) The single-scattering albedo of cloud droplet in the $2.1-\mu \mathrm{m}$ (blue) and $3.7-\mu \mathrm{m}$ (red) band, and the asymmetry factor in the $0.86-\mu \mathrm{m}$ band (black) plotted as a function of cloud droplet radius. The dashed lines indicate polynomial fitting to the single-scattering albedos based on Eq. (4.7). (b) The bulk single-scattering albedo in the 2.1- $\mu \mathrm{m}$ (blue) and 3.7- $\mu \mathrm{m}$ (red) band, and the asymmetry factor in the $0.86-\mu \mathrm{m}$ band (black) plotted as a function of cloud droplet effective radius. The dashed lines indicate polynomial fitting to the single-scattering albedos based on Eq. (4.8). In the computation, monomodal Gamma size distribution with $v_{e}=0.1$ is assumed.

the asymptotic value of 0.5 . Thus, the curvature of the relationship between $\omega_{\lambda}$ and $r$ results in the negative value of the $k_{2, \lambda} / \bar{k}_{1, \lambda}$ term. The magnitude of this term is determined by the ratio $\left\langle r^{4}\right\rangle /\left\langle r^{2}\right\rangle$ which can be shown to be equivalent to $r_{e}^{2}\left(1+v_{e}\right)$. This relationship reveals that the impact of the second-order term depends on $r_{e}$ and to a much lesser extent on $v_{e}$, namely the width of the cloud DSD. This relationship also implies that, in order for the nonlinear term to significantly affect $\bar{\omega}_{\lambda}$, both $r_{e}$ and $v_{e}$ have to be sufficiently large. Interestingly, such conditions happen to be favored in precipitating clouds, as they tend to have larger $r_{e}$ and precipitating processes (e.g. collision-coalescence) tend to broaden the DSD (i.e. increase $v_{e}$ ) (Berry, 1967; Berry and Reinhardt, 1974; Pruppacher and Klett, 1997).

Focusing now on the spectral dependence of Eq. (4.9), it is seen from Table 4.1 that the magnitude of $k_{i, 2.1 \mu m} / \bar{k}_{1,2.1 \mu \mathrm{m}}$ is substantially smaller than $k_{i, 3.7 \mu \mathrm{m}} / \bar{k}_{1,3.7 \mu \mathrm{m}}$. It indicates a stronger impact of the nonlinear terms on $r_{e}$ retrieval in the $3.7-\mu \mathrm{m}$ band than the $2.1-\mu \mathrm{m}$ band. For the $r_{e, 2.1 \mu \mathrm{m}}^{*}$ retrieval, only the second-order nonlinear term is significant. But, for the $r_{e, 3.7 \mu m}^{*}$ retrieval, higherorder terms need also to be included, although the second-order term is still the dominant nonlinear term. As discussed above, the second-order nonlinear term tends to result in underestimated $r_{e}^{*}$ retrieval. This underestimation is expected to be stronger in the $3.7-\mu \mathrm{m}$ band than in the $2.1-\mu \mathrm{m}$ band, resulting in negative $r_{e, 3.7 \mu m}^{*}-r_{e, 2.1 \mu m}^{*}$. This theoretical prediction is consistent with the numerical results in Minnis et al. (2004) and Nakajima et al. (2010a). It was found in these studies that increasing rain mode in a bi-modal DSD leads to underestimated $r_{e}$ retrieval and the underestimation is more severe in the $3.7-\mu \mathrm{m}$ band than the $2.1-\mu \mathrm{m}$ band.

In summary, Zhang (2013) illustrated that $r_{e}$ retrieval-based monomodal DSD assumption tends to underestimate the $r_{e}$ of bi-modal DSD, due to the nonlinear 
relationship between cloud droplet single-scattering albedo and cloud droplet size. The underestimation is stronger for $r_{e, 3.7 \mu m}$ than for $r_{e, 2.1 \mu m}$, leading to a negative $\Delta r_{e, 3.7-2.1}$.

\subsubsection{Plane-parallel $r_{e}$ bias}

As mentioned in section 4.2, one of the fundamental assumptions made in the MODIS retrieval algorithm is that a cloudy $1 \mathrm{~km} \times 1 \mathrm{~km}$ pixel is horizontally homogenous. However, it is known that clouds can have significant horizontal variability at smaller scales, leading to sub-pixel inhomogeneity (e.g. Davis et al., 1994; Marshak et al., 1995). When the (horizontally) homogeneous pixel assumption breaks down, both MODIS $\tau$ and $r_{e}$ retrievals face challenging issues. For $\tau$ retrieval, for example, sub-pixel inhomogeneity can cause the well-known planeparallel albedo bias. That is, the $\tau$ retrieval based on the average reflectance of a heterogeneous cloudy pixel tends to be smaller than the average of the sub-pixel scale $\tau$ (e.g. Cahalan et al., 1994; Barker, 1996; Oreopoulos and Davies, 1998). Sub-pixel inhomogeneity also affects $r_{e}$ retrieval in various ways, one of which is the so-called plane-parallel $r_{e}$ bias (Zhang and Platnick, 2011; Zhang et al., 2012). Plane-parallel $r_{e}$ bias tends to result in overestimation of $r_{e}$ and it affects the $r_{e 2.1}$ retrieval more than $r_{e 3.7}$, therefore leading to spectral difference (negative $\Delta r_{e, 3.7-2.1}$ ) (Zhang and Platnick, 2011; Zhang et al., 2012).

The plane-parallel $r_{e}$ bias is defined as the impact of small-scale variability in $\tau$ on $r_{e}$ retrievals that use area-averaged reflectance (Zhang and Platnick, 2011; Zhang et al., 2012). This bias is illustrated using two idealized examples in Fig. 4.9, which shows forward calculations of reflectances in $0.86-\mu \mathrm{m}, 2.1-\mu \mathrm{m}$ and $3.7-\mu \mathrm{m}$ bands. In Fig. 4.9a and 4.9b, we assume that half of a MODIS pixel overlying a black surface is covered by a cloud with $\tau_{1}=2.8$ and $r_{e}=8 \mu \mathrm{m}$ and the other half is covered by a cloud with $\tau_{2}=30.8$ and $r_{e}=8 \mu \mathrm{m}$. Panels c and d assume the same optical thickness but use $r_{e}=18 \mu \mathrm{m}$. Focusing on the $\tau$ retrieval, the figure illustrates the well-known 'plane-parallel albedo bias' (Cahalan et al., 1994): the retrieved $\tau$ based on the mean reflectance of inhomogeneous pixels tends to be smaller than the mean of the sub-pixel $\tau$. In this example the value of retrieved from the averaged reflectance $(\tau=10.8)$ is substantially smaller than the average value $(\bar{\tau}=16.8)$.

This problem is more acute for retrievals of $r_{e}$ because the reflectance used to infer $r_{e}$ also depends on $\tau$ over much of the range of plausible values, as has been shown in section 4.4.1. If the reflectance at non-absorbing and absorbing wavelengths depended only on $\tau$ and $r_{e}$, respectively (i.e. if the LUT was orthogonal), reflectance at absorbing wavelengths would be uniform in our example and particle size could be retrieved perfectly. As Fig. 4.9 demonstrates, however, the LUT is not orthogonal. The nonlinearity leads to a simultaneous underestimation of $\tau$ (plane-parallel-albedo bias) and overestimation of $r_{e}$ (plane-parallel $r_{e}$ bias). The area over which this is true is larger in the less-absorbing band, which explains why $r_{e, 3.7}$ is smaller than $r_{e, 2.1}$. The impact becomes more pronounced as $r_{e}$ increases: in Fig. $4.9 \mathrm{c}$ and $4.9 \mathrm{~d}$, the true $r_{e}=18 \mu \mathrm{m}$ while $r_{e, 2.1}$ and $r_{e, 3.7}$ retrieved from averaged reflectances are $24 \mu \mathrm{m}$ and $18.1 \mu \mathrm{m}$, respectively, resulting in a $\Delta r_{e, 3.7-2.1}$ around $-6 \mu \mathrm{m}$. 

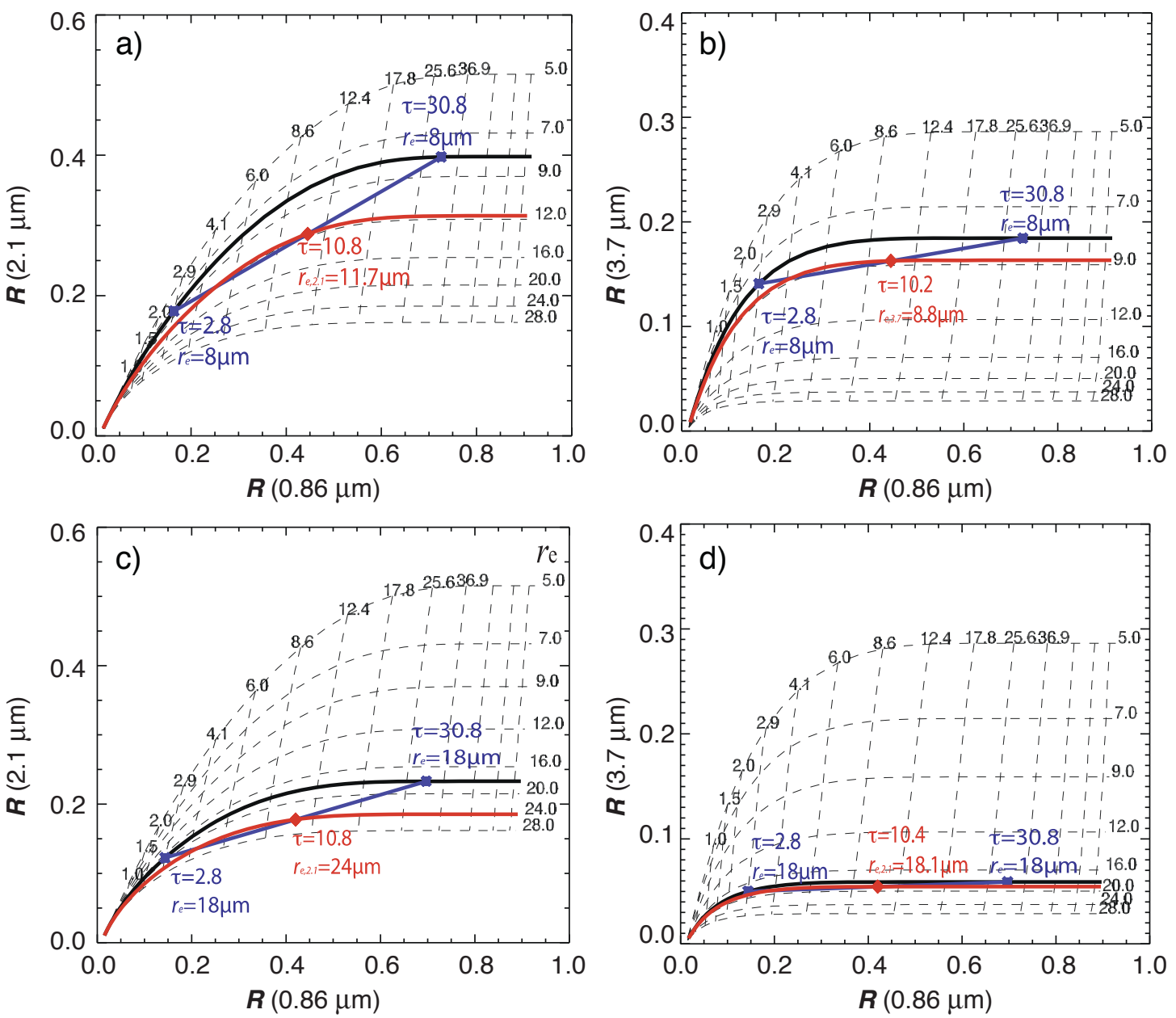

Fig. 4.9. Two theoretical cases to illustrate the nonlinearity effect in $r_{e}$ retrievals resulting from sub-pixel cloud inhomogeneity. Numbers on top of the Nakajima-King look-up-table (LUT) curves correspond to values of $\tau$ contour lines in the LUT and the numbers on the right of the curves correspond to values of $r_{e}$ contour lines in the LUT.

Recently, Zhang et al. (2012) developed a numerical MODIS retrieval simulator based on the combination of an LES model with bin microphysics (Ackerman et al., 2004; Fridlind and Ackerman, 2010) and radiative transfer models (both 1D and $3 \mathrm{D})$. In the case study based on this simulator, they confirmed that the $r_{e}$ planeparallel bias, as conceptually illustrated in Fig. 4.9, indeed play an important role in causing the spectral difference between $r_{e, 2.1}$ and $r_{e, 3.7}$, especially for those pixels with high sub-pixel inhomogeneity.

As shown earlier in Fig. 4.5a, the statistics of MODIS $r_{e, 2.1}$ show a strong dependence on the sub-pixel inhomogeneity index, $H_{\sigma}$. When a cloud is relatively homogeneous (i.e. $H_{\sigma}<0.3$ ) the peak of the PDF of MODIS $r_{e, 2.1}$ is around $12 \sim 14 \mu \mathrm{m}$. When $H_{\sigma}$ becomes larger than about 0.3 , the peak increases quickly with $H_{\sigma}$, by up to about $20 \mu \mathrm{m}$ when $H_{\sigma}$ is about unity. Interestingly, MODIS $r_{e, 3.7}$ shows little dependence in Fig. 4.5b, leading to significant negative $\Delta r_{e, 3.7-2.1}$ when $H_{\sigma}>0.3$. The behaviors of $r_{e, 2.1}, r_{e, 3.7}$ and $\Delta r_{e, 3.7-2.1}$ in Fig. 4.5 are consistent with the concept of $r_{e}$ plane-parallel bias as shown Fig. 4.9, therefore suggesting 
$r_{e}$ plane-parallel bias is an important factor causing the $\Delta r_{e, 3.7-2.1}$. On the other hand, Fig. 4.5 also suggests that the impact of $r_{e}$ plane-parallel bias is limited to those highly inhomogeneous pixels, with $H_{\sigma}$ larger than about 0.3 .

\subsection{5 $3 \mathrm{D}$ radiative transfer effect}

As shown in a couple of recent studies (Zhang and Platnick, 2011; Zhang et al., 2012), 3D radiative transfer effects generally influence $r_{e, 2.1}$ and $r_{e, 3.7}$ retrievals to different extents. This makes $3 \mathrm{D}$ radiative transfer a potentially important factor in causing the spectral dependence of MODIS $r_{e}$ retrieval as described in section 4.3.

As mentioned in section 4.2, the MODIS retrieval algorithm assumes the socalled plane-parallel cloud model. However, clouds in reality have significant horizontal structures and variabilities at various scales, from thousands of kilometers to a few meters (Cahalan et al., 1994; Davis et al., 1994; Wood and Hartmann, 2006; Wood et al., 2008). Horizontal cloud variability gives rise to horizontal photon transport (Davis and Marshak, 2010), which makes the observed reflectance dependent on not only the property of pixels within the field of view, but also the properties of surroundings (Marshak et al., 1995). In such circumstances, cloud properties retrieved under the plane-parallel cloud assumption are subject to significant errors. This is the so-called 3D radiative transfer effect. Take the so-called 'shadowing effect', for example (Várnai and Marshak, 2002; Marshak et al., 2006). Consider, for example, a cloudy pixel that is in the shadow of an adjacent pixel. The darkening of the pixel would be fallaciously interpreted under the plane-parallel cloud assumption, leading to underestimated $\tau$ and overestimated $r_{e}$ retrievals (Várnai and Marshak, 2002; Marshak et al., 2006; Zhang and Platnick, 2011; Zhang et al., 2012).

It was shown in a couple of recent studies (Zhang and Platnick, 2011; Zhang et al., 2012) that 3D radiative transfer effects generally have a stronger impact on the $r_{e, 2.1}$ retrieval than on the $r_{e, 3.7}$ retrieval. For example, when the shadowing effect leads to an overestimation of $r_{e}$, the magnitude of overestimation is larger for $r_{e, 2.1}$ than for $r_{e, 3.7}$. The reason for this is two-fold. First, 3D radiative transfer effect results from horizontal photon transport and the strength of droplet absorption in the 3.7- $\mu \mathrm{m}$ band exerts a stronger limit on horizontal photon transport than the weaker 2.1- $\mu \mathrm{m}$ band absorption. Secondly, as shown in sections 4.4.1 and 4.4.4, the LUT for the $0.86-\mu \mathrm{m}$ and $2.1-\mu \mathrm{m}$ combination is less orthogonal than that for the $0.86-\mu \mathrm{m}$ and $3.7-\mu \mathrm{m}$ combination. As a consequence, the $r_{e, 2.1}$ retrieval is more sensitive to $3 \mathrm{D}$ radiative transfer effects on the $0.86-\mu \mathrm{m}$ band reflectance than $r_{e, 3.7}$ retrieval.

Recently, Zhang et al. (2012) studied the impact of 3D radiative effects, as well as other factors, on MODIS cloud property retrievals using an LES model with a binned microphysical scheme (Ackerman et al., 2004; Fridlind and Ackerman, 2010) coupled with a 3D radiative transfer model (Cahalan et al., 2005; Pincus and Evans, 2009). Figure 4.10 shows one of the LES cases in this study, which is based on an idealized case study (Stevens et al., 2001) from the Atlantic Trade Wind Experiment (ATEX), with an average cloud droplet concentration (weighted by liquid water mixing ratio) of about $30 \mathrm{~cm}^{-3}$. For more details about this LES case and the configurations of radiative transfer models, readers are referred to Zhang 
et al. (2012). Figure 4.10a provides a planar view of the cloud $\tau$ derived from the LES for this case. Figure 4.10b shows the cloud extinction coefficient along the vertical cross-section indicated by the red dashed line in Fig. 4.10a. In radiative transfer simulations, solar zenith angle is assumed to be $20^{\circ}$ and viewing zenith angle to be $0^{\circ}$. The $1 \mathrm{D}$ and $3 \mathrm{D}$ simulations of cloud reflectances along the crosssection for the $0.86-\mu \mathrm{m}, 2.1-\mu \mathrm{m}$ and $3.7-\mu \mathrm{m}$ bands are shown in Figs. 4.10c, 4.10d and $4.10 \mathrm{e}$, respectively. Although 1D and 3D simulations are in general agreement, the influence of horizontal photon transport can be clearly seen in a few regions, especially in Fig. 4.10c. For example, at $x=7 \mathrm{~km}$ the $0.86-\mu \mathrm{m}$ cloud reflectance based on 1D simulation is substantially larger than 3D simulation. But, on both sides of this reflectance peak (i.e. $x=6.5 \mathrm{~km}$ and $x=7.5 \mathrm{~km}$ ), $3 \mathrm{D}$ reflectance is larger than 1D. These differences between 1D and 3D simulations are the result of horizontal photon transport due to multiple scattering (Várnai and Davies, 1999). Interestingly, this horizontal photon transport effect is much reduced in the $2.1-\mu \mathrm{m}$ and 3.7- $\mu \mathrm{m}$ simulations. This is because the absorption in the SWIR bands limits multiple scattering and therefore reduces the horizontal transport of photons.

Figure 4.11 shows the impacts of $3 \mathrm{D}$ radiative transfer effects on $r_{e, 2.1}$ and $r_{e, 3.7}$ for the case in Fig. 4.10. In the figure, we use the difference between the $\tau$ retrieval based on $3 \mathrm{D}$ radiative transfer simulation (referred to as ' $3 \mathrm{D} \tau$ ') and that based on $1 \mathrm{D}$ radiative transfer simulation (referred to as ' $1 \mathrm{D} \tau$ ') as an index of the 3D radiative transfer effects. By definition, 3D $\tau>1 \mathrm{D} \tau$ in the case of illumination, and 3D $\tau<1 \mathrm{D} \tau$ in the case of shadowing. As expected, the $r_{e}$ retrievals based on $3 \mathrm{D}$ radiative transfer simulation ('3D $r_{e}$ ') appear smaller than those based on $1 \mathrm{D}$ radiative transfer simulation ('1D $r_{e}$ ') in the case of illuminating effect (i.e. 3D $r_{e}-1 \mathrm{D} r_{e}<0$ ), and vice versa in the case of shadowing effect (i.e. $\left.3 \mathrm{D} r_{e}-1 \mathrm{D} r_{e}>0\right)$. What is interesting in Fig. 4.11 is that the impact of 3D radiative effects on $r_{e, 2.1}$ is generally stronger than on $r_{e, 3.7}$, for the reasons discussed earlier in this section. Another important point to note in Fig. 4.11 is that illuminating and shadowing effects naturally come in pairs and tend to cancel each other out (Marshak et al., 2006). Therefore, 3D radiative effects seem unlikely to cause large systematic bias in $r_{e}$ retrieval. Moreover, since 3D radiative effects are a result of horizontal photon transport, its impact on MODIS retrievals is limited to pixels with large horizontal inhomogeneity. In addition, when a cloud pixel has strong horizontal inhomogeneity, both $3 \mathrm{D}$ radiative effects and the plane-parallel $r_{e}$ bias discussed in section 4.4.4 may play a role at the same time, creating either positive or negative $\Delta r_{e, 3.7-2.1}$ (Zhang et al., 2012).

\subsection{Discussion}

In the previous section, we reviewed several mechanisms that could cause significant difference between MODIS $r_{e, 2.1}$ and $r_{e, 3.7}$ retrievals for MBL clouds. But the most fundamental reason is that MBL clouds in reality do not always satisfy the ideal cloud model (e.g. plane-parallel and homogenous) on which MODIS is based. When the cloud deviates from the ideal model, the deviation affects the $r_{e, 2.1}$ and $r_{e, 3.7}$ retrievals to different extents or in different ways, leading to significant difference between the two. From this perspective, spectral dependence is an inherent and 

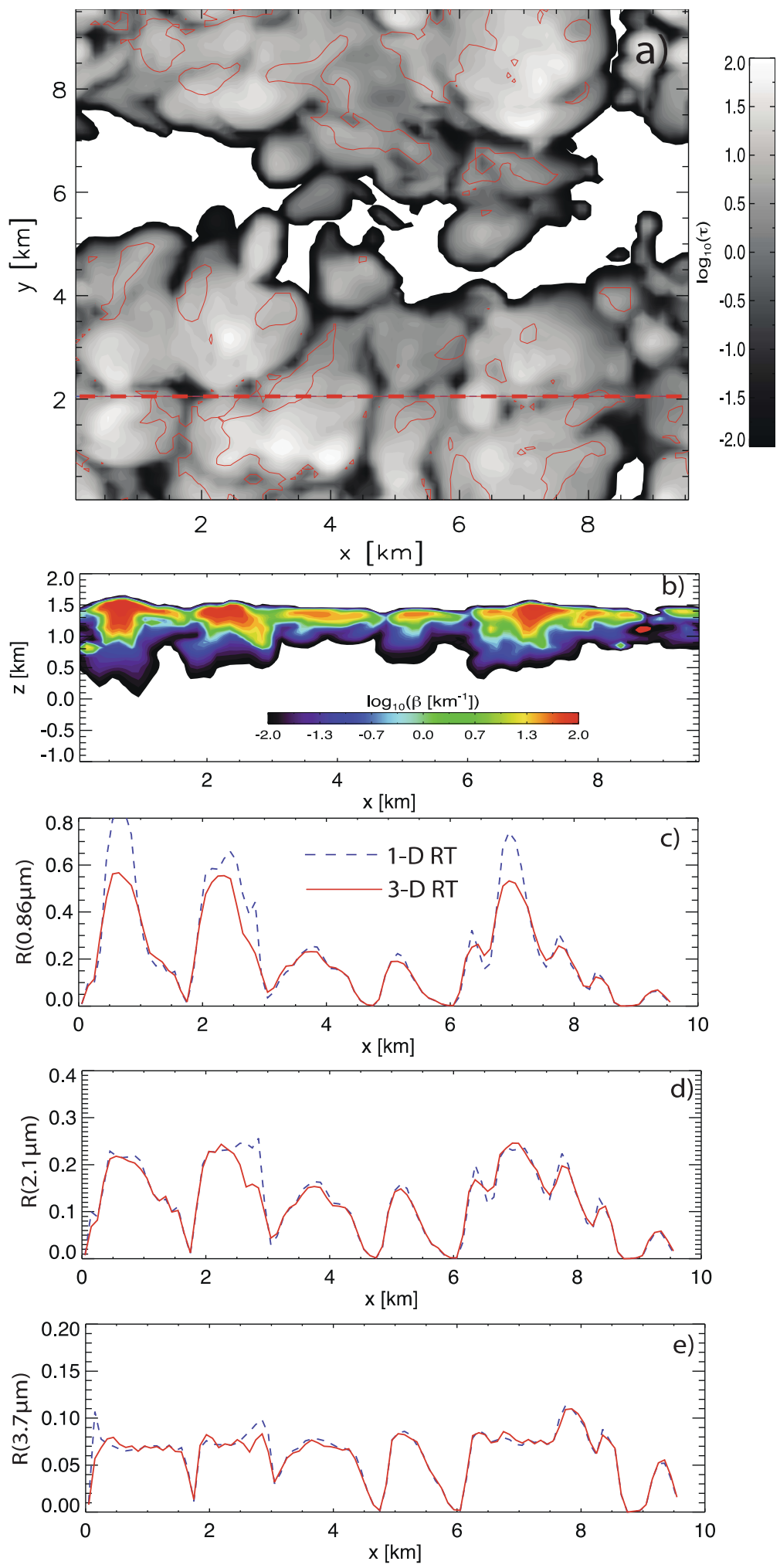

Fig. 4.10. (a) Planar view of the cloud $\tau$ of the ATEX clean case at $6 \mathrm{~h}$ of simulation time. (b) The cross-section of cloud extinction coefficient $(\beta)$ along $y=2 \mathrm{~km}$ in (a). Cloud bi-directional reflectance along the cross section is shown for the (c) $0.86-\mu \mathrm{m}$, (d) $2.1-\mu \mathrm{m}$, and (e) 3.7- $\mu \mathrm{m}$ MODIS bands simulated using 1D (blue) and 3D (red) radiative transfer models. 

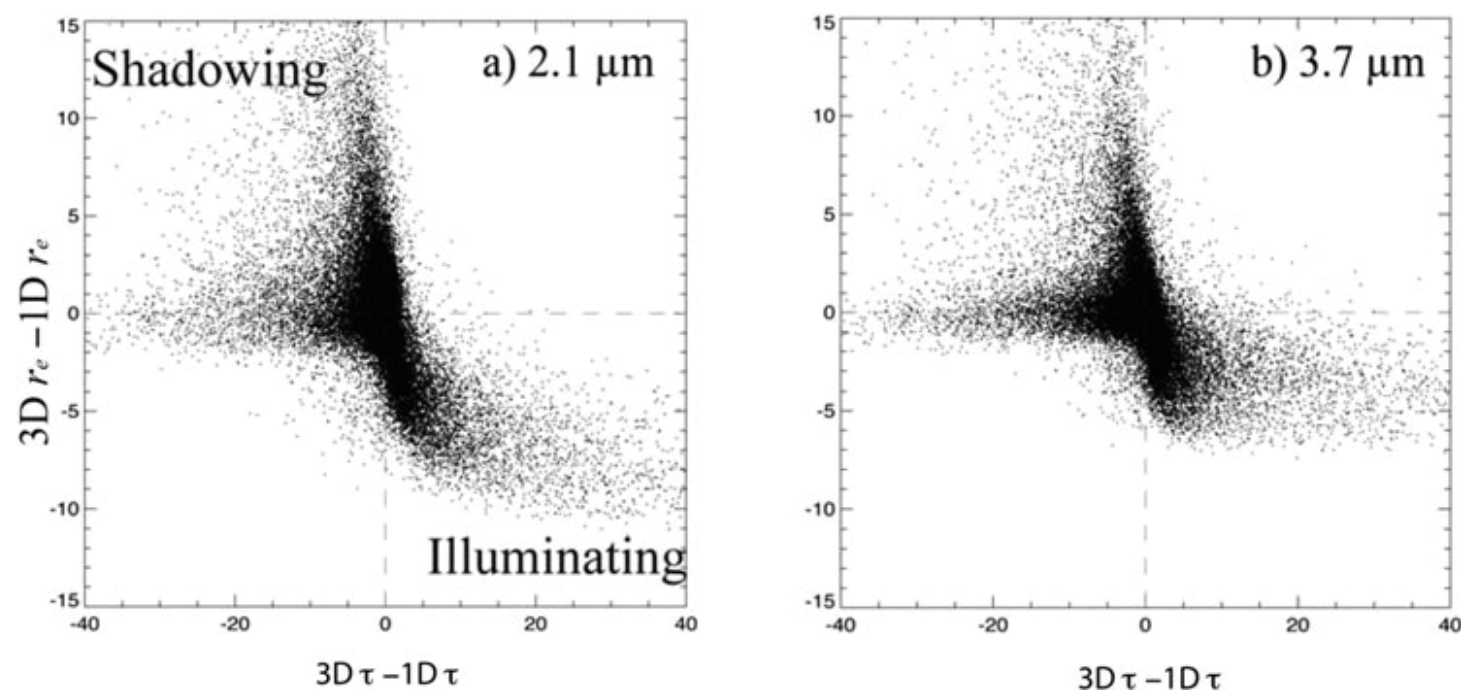

Fig. 4.11. The difference between $3 \mathrm{D}$ and $1 \mathrm{D} \tau$ retrieval vs. the difference between (a) 3D $r_{e, 2.1}$ and 1D $r_{e, 2.1}$ at LES resolution, (b) 3D $r_{e, 3.7}$ and 1D $r_{e, 3.7}$ at LES resolution.

inevitable feature of the $r_{e}$ retrieval based on the bi-spectral method. On one hand, it reflects the limitations of the method but, on the other, it indicates that the operational MODIS retrieval results are generally consistent with our expectations.

\subsubsection{Which one is better?}

If spectral dependence is inevitable in MODIS $r_{e}$ retrieval, then a natural question arises: which one, $r_{e, 2.1}$ or $r_{e, 3.7}$, is better or more correct? Unfortunately, there is no simple answer to this question. As shown in section 4.4.1, $r_{e, 3.7}$ is less affected by random retrieval uncertainties, if uncertainties associated with $2.1-\mu \mathrm{m}$ and 3.7$\mu \mathrm{m}$ band observations are of similar magnitude. Also, in comparison with $r_{e, 2.1}$, $r_{e, 3.7}$ is generally less affected by the plane-parallel $r_{e}$ and $3 \mathrm{D}$ radiative effects as discussed in sections 4.4.4 and 4.4.5. In these cases, $r_{e, 3.7}$ is expected to perform better than $r_{e, 2.1}$. However, as discussed in section 4.4.2, from the perspective of vertical weighting, $r_{e, 2.1}$ and $r_{e, 3.7}$ simply reflect different parts of the cloud. Simply put, there is no right or wrong. Moreover, as discussed in section 4.4.3, when the true cloud DSD is bi-modal, both $r_{e, 2.1}$ and $r_{e, 3.7}$ tend to underestimate the true $r_{e}$, but the underestimation is more severe in the $r_{e, 3.7}$ than in the $r_{e, 2.1}$. Therefore, whether to use $r_{e, 2.1}$ or $r_{e, 3.7}$ depends on the intended application and the geographical location of interest. For example, several studies have attempted to retrieve the vertical profile of MBL clouds utilizing the combination of $r_{e, 2.1}$ and $r_{e, 3.7}$ retrievals from MODIS (Chang and Li, 2002, 2003; Kokhanovsky and Rozanov, 2011). For these applications, both $r_{e, 2.1}$ and $r_{e, 3.7}$ are needed and their difference serves as a useful signal rather than a data issue. As shown in Fig. 4.2, $r_{e, 2.1}$ and $r_{e, 3.7}$ agree quite well over the coastal stratocumulus regions. So, if those regions are of interest, it perhaps makes little difference which retrieval is used. In contrast, over broken cloud regions where clouds have significant horizontal heterogeneity, $r_{e, 3.7}$ would be less affected by plane-parallel $r_{e}$ bias and 3D radiative effect and therefore might be a better choice than $r_{e, 2.1}$. 
It should be noted here that $r_{e, 3.7}$ is reported as having a respective difference to successful $r_{e, 2.1}$ retrieval in the Collection 5 of MODIS cloud product. Thus, in Collection 5, the sampling of $r_{e, 3.7}$ is biased by the success of $r_{e, 2.1}$. In addition, although retrieved in Level 2 products, $r_{e, 3.7}$ is not aggregated to Level 3 products. In the current Collection 6 of MODIS cloud product, these sampling biases will be removed and $r_{e, 3.7}$ will be sampled independently and aggregated to Level 3 data. These changes will facilitate the use of $r_{e, 3.7}$.

\subsubsection{Cloud regime classification}

Summarizing the discussions in the previous section, one can see that none of the hypotheses can uniquely explain all of the $\Delta r_{e, 3.7-2.1}$ features shown in section 4.3. This is surprising because all these mechanisms are more or less entangled together in the retrieval. For example, when a cloud pixel has strong horizontal inhomogeneity, both the plane-parallel $r_{e}$ bias (section 4.4.4) and 3D radiative effects (section 4.4.5) may play a role at the same time. When warm rain processes begin to develop in MBL clouds, it can lead to both cloud vertical structure and bi-model DSD, in which case both the vertical weighting effect (section 4.4.2) and the DSD sensitivity effect (section 4.4.3) will influence MODIS $r_{e}$ retrievals. Thus, for a better understanding, we need to untangle these mechanisms and sort out their relative importance in different cloud regimes. By 'cloud regimes', we mean groups of MBL clouds with different characteristics in terms of optical (e.g. $\tau$ ), microphysical (e.g. $r_{e}$ ), and macrophysical (e.g. horizontal properties). An attempt of such cloud regime classification and analysis has been made in Fig. 4.12. It shows a color contour of the mean value of $\Delta r_{e, 3.7-2.1}$ projected on sub-pixel heterogeneity index $H_{\sigma}$ and $r_{e, 2.1}$ for MBL clouds with $\tau>5$. The black contour lines indicate the joint frequency histogram of $H_{\sigma}$ and $r_{e, 2.1}$ based on one month of MODIS observation. Based on the combination of $r_{e, 2.1}$ and $H_{\sigma}$, MBL cloud pixels observed by MODIS can be classified into the following three regimes:

- Regime 1: MBL cloud pixels in this regime have low horizontal heterogeneity $\left(H_{\sigma}<0.3\right)$ and relatively small effective radius $\left(r_{e, 2.1}<20 \mu \mathrm{m}\right)$. Low $H_{\sigma}$ index suggests that the plane-parallel $r_{e}$ bias and 3D radiative effects should be small in this regime. Small $r_{e, 2.1}$ suggests that the probability of warm rain precipitation is relatively small because the critical value of $r_{e}$ for collisioncoalescence to occur is around $15 \mu \mathrm{m}$ (Gerber, 1996; Rosenfeld et al., 2012). As a result, the vertical weighting effect and the DSD sensitivity effect can be expected to be small. It now becomes clear that MBL cloud pixels in this regime, with low horizontal heterogeneity and low possibility of precipitation, satisfy those assumptions made in MODIS cloud retrieval mentioned in section 4.2. For this reason, it is not surprising to see good agreement between $r_{e, 2.1}$ and $r_{e, 3.7}$ in this regime $\left(\Delta r_{e, 3.7-2.1}\right.$ is within $\left.\pm 2 \mu \mathrm{m}\right)$, although a small difference, probably due to random retrieval uncertainties, may still exist.

- Regime 2: MBL cloud pixels in this regime are characteristic with high horizontal heterogeneity $\left(H_{\sigma}>0.3\right)$. Retrieval uncertainties, plane-parallel $r_{e}$ bias, and $3 \mathrm{D}$ radiative effects may all play a role in this regime. Note that these effects have different impacts on $r_{e}$ retrievals and therefore $\Delta r_{e, 3.7-2.1}$. Retrieval uncertainties, as discussed in section 4.4 .1 , are most likely to cause random 


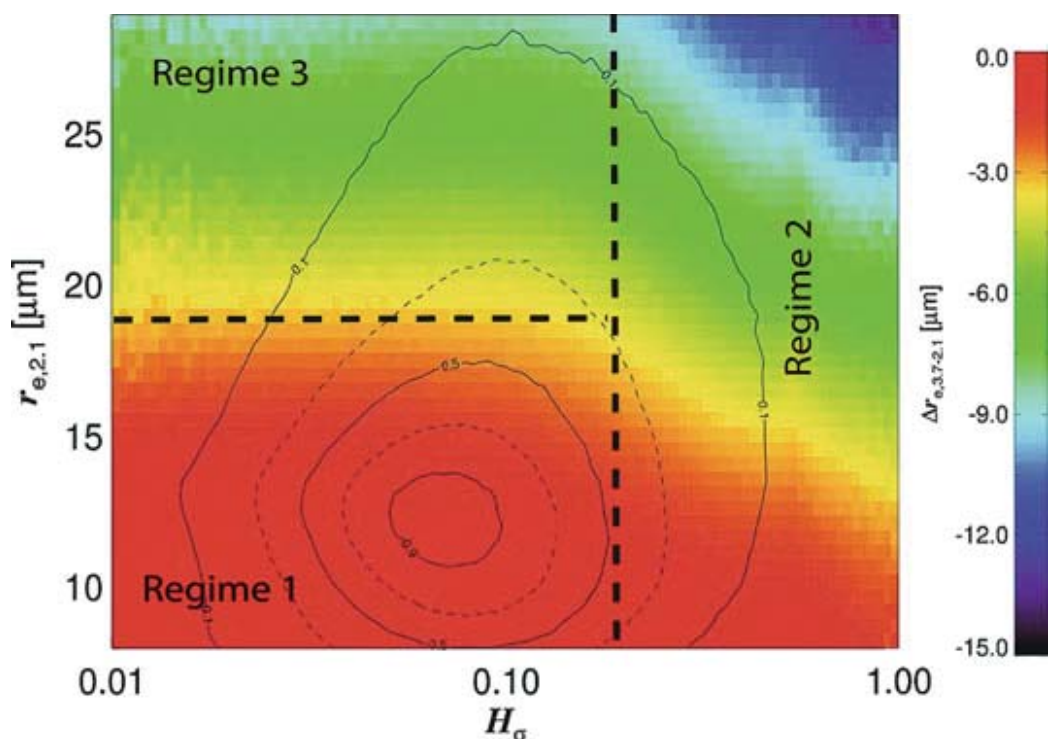

Fig. 4.12. The color contour of monthly mean $\Delta r_{e, 3.7-2.1}$ for clouds with $\tau>5$ on the space specified by $r_{e, 2.1}$ and $H_{\sigma}$. The black lines indicate the relative frequency of each grid box, specified by certain combinations of $r_{e, 2.1}$ and $H_{\sigma}$ (unity corresponds to the most frequently observed combination of $r_{e, 2.1}$ and $H_{\sigma}$ ). See text for details on cloud regime classification and implications for MODIS effective radius retrievals.

errors, rather than bias, in $\Delta r_{e, 3.7-2.1}$. Similarly, as discussed in section 4.4.5, $3 \mathrm{D}$ radiative effects can cause either positive or negative $\Delta r_{e, 3.7-2.1}$, depending on the nature (e.g. illuminating or shadow) of the 3D effect. In contrast, the plane-parallel $r_{e}$ bias, as discussed in section 4.4.4, tends to cause negative $\Delta r_{e, 3.7-2.1}$.

- Regime 3: MBL cloud pixels in this regime have low horizontal heterogeneity $\left(H_{\sigma}<0.3\right)$ and relatively large effective radius $\left(r_{e, 2.1}>20 \mu \mathrm{m}\right)$. Large effective radius suggests that MBL clouds in this regime are possibly precipitating. The warm rain process can give rise to the vertical weighting effect and the DSD sensitivity effect, as discussed in section 4.4.2 and 4.4.3 respectively. Because both effects tend to make $r_{e, 2.1}$ larger than $r_{e, 3.7}$, thus the bias of this regime is large and negative, as seen in Fig. 4.12.

\subsection{Outlook of future work}

Recently, the spectral dependence of MODIS $r_{e}$ retrievals for MBL clouds has received significant and increasing attention. A number of recent studies reviewed in this chapter have significantly improved our understanding of the nature and potential causes of this issue. Nevertheless, many outstanding questions remain and future work is needed particularly in the following areas: (i) More quantitative understanding: Together, those hypotheses reviewed in section 4.4 provide a qualitative explanation for the $r_{e, 2.1}$ and $r_{e, 3.7}$ differences. However, a more quantitative understanding is still lacking. It remains unclear whether the threshold behavior of $\Delta r_{e, 3.7-2.1}$ at $r_{e, 2.1} \sim 15 \mu \mathrm{m}$ in Fig. 4.3 is a coincidence or a result of warm rain 
process. In order to answer questions like this, hypotheses must be evaluated in a more quantitative way in future work. (ii) Independent measurements: For more objective and quantitative evaluation of MODIS $r_{e, 2.1}$ and $r_{e, 3.7}$ retrievals, independent measurements are needed. For example, several studies have investigated the impact of 3D radiative effects on cloud masking (Zhao and Di Girolamo, 2006), cloud optical thickness, and cloud effective radius retrievals (Marshak et al., 2006) using the high spatial resolution ASTER (Advanced Spaceborne Thermal Emission and Reflection Radiometer) observations. The ASTER observation may prove useful in future analyses to understand the relative importance of the impacts of $3 \mathrm{D}$ radiative effects and plane-parallel $r_{e}$ retrieval bias on cloud property retrievals. Several studies have demonstrated that high temporal-spatial resolution cloud DSD measurements from air-borne in situ instruments, collocated with MODIS observation, are very helpful for understanding the errors and uncertainties in MODIS retrievals. Other remote sensing measurements, in particular those from A-Train satellite sensors (e.g. CloudSat, POLDER, and CALIPSO), would add unique and valuable perspectives on MBL cloud microphysics that are worthy of exploration in future work. (iii) Combining observations with numerical models: Every remote sensing method has its limitations. Even though a combination of various methods, such as those available from A-Train sensors, provides a complementary perspective, there will always be gaps that cannot be covered by observations. As shown in a number of recent studies, a combination of LES models with satellite retrieval simulators provides a flexible and powerful tool for understanding the impact of various factors on MODIS cloud property retrievals (e.g. Zinner et al., 2010; Zhang et al., 2012). This new avenue of research should be further explored in future work. (iv) Implication studies: MODIS cloud products are popularly used in climate change studies, aerosol indirect effect studies, and climate model validations. The implications of the spectral dependence of MODIS $r_{e}$ retrievals for these 'down-stream' applications should be investigated in future work. (v) Novel use of $r_{e, 2.1}$ and $r_{e, 3.7}$ : As discussed in sections 4.4.2 and 4.4.3, in certain circumstances, the difference between $r_{e, 2.1}$ and $r_{e, 3.7}$ actually contains useful information about MBL clouds. Actually, a number of studies have attempted to retrieve the vertical profile of precipitating MBL clouds utilizing the combination of effective radius retrievals from different spectral bands (Chang and Li, 2002, 2003; Kokhanovsky and Rozanov, 2011). Further work is needed along these lines to fully explore the potential of MODIS observations.

\section{Acknowledgments}

The authors acknowledge funding from NASA through grant NNX11AI98G managed by Dr. Richard Eckmann. Z.Z.'s research is also supported by the start-up funding from UMBC. Computational support was provided by the NASA Advanced Supercompting Division. 


\section{References}

Ackerman, A.S., Kirkpatrick, M.P., Stevens, D.E. and Toon, O.B. (2004) 'The impact of humidity above stratiform clouds on indirect aerosol climate forcing', Nature, 432, $1014-17$.

Austin, R.T., Heymsfield, A.J. and Stephens, G.L. (2009) 'Retrieval of ice cloud microphysical parameters using the CloudSat millimeter-wave radar and temperature', $J$. Geophys. Res., 114, D00A23, doi:10.1029/2008JD010049.

Baker, M.B., Corbin, R.G. and Latham, J. (1980) 'The influence of entrainment on the evolution of cloud droplet spectra: I. A model of inhomogeneous mixing', Quarterly Journal of the Royal Meteorological Society, 106, 581-98.

Barker, H.W. (1996) 'A parameterization for computing grid-averaged solar fluxes for inhomogeneous marine boundary layer clouds: Part I: Methodology and homogeneous biases', J. Atmos. Sci., 53, 2289-303.

Berry, E.X. (1967) 'Cloud droplet growth by collection', J. Atmos. Sci., 24, 688-701.

Berry, E.X. and Reinhardt, R.L. (1974) 'An analysis of cloud drop growth by collection: Part I: Double distributions', Journal of Atmospheric Sciences, 31, 1814-24.

Brenguier, J.-L., Pawlowska, H., Schüller, L., Preusker, R. and co-authors (2000) 'Radiative properties of boundary layer clouds: Droplet effective radius versus number concentration', J. Atmos. Sci., 57, 803-21.

Breon, F. and Doutriaux-Boucher, M. (2005) 'A comparison of cloud droplet radii measured from space', IEEE Transactions on Geoscience and Remote Sensing, 43, 1796.

Cahalan, R.F., Oreopoulos, L., Marshak, A., Evans, K.F., Davis, A.B. and co-authors (2005) 'THE I3RC: Bringing together the most advanced radiative transfer tools for cloudy atmospheres', Bull. Amer. Meteor. Soc., 86, 1275-93.

Cahalan, R.F., Ridgway, W., Wiscombe, W.J., Bell, T.L. and Snider, J.B. (1994) 'The albedo of fractal stratocumulus clouds', J. Atmos. Sci., 51, 2434-55.

Chang, F.L. and Li, Z. (2001) 'The effect of droplet size distribution on the determination of cloud droplet effective radius', in Proceedings of the 11th ARM Science Team Meeting, Atlanta, GA.

Chang, F.L. and Li, Z. (2002) 'Estimating the vertical variation of cloud droplet effective radius using multispectral near-infrared satellite measurements', J. Geophys. Res., $107,4257$.

Chang, F.L. and Li, Z. (2003) 'Retrieving vertical profiles of water-cloud droplet effective radius: Algorithm modification and preliminary application', J. Geophys. Res., 108, 4763 .

Chen, R., Chang, F.L., Li, Z., Ferraro, R. and Weng, F. (2007) 'Impact of the vertical variation of cloud droplet size on the estimation of cloud liquid water path and rain detection', J. Atmos. Sci., 64, 3843-53.

Davis, A., Marshak, A., Wiscombe, W. and Cahalan, R. (1994) 'Multifractal characterizations of nonstationarity and intermittency in geophysical fields: Observed, retrieved, or simulated', Journal of Geophysical Research-All Series, 99, 8055.

Davis, A.B. and Marshak, A. (2010) 'Solar radiation transport in the cloudy atmosphere: A 3D perspective on observations and climate impacts', Reports on Progress in Physics, 73, 026801.

Di Girolamo, L., Liang, L. and Platnick, S. (2010) 'A global view of one-dimensional solar radiative transfer through oceanic water clouds', Geophys. Res. Lett., 37, L18809.

Fridlind, A.M. and Ackerman, A.S. (20100 'Estimating the sensitivity of radiative impacts of shallow, broken marine clouds to boundary layer aerosol size distribution parameter uncertainties for evaluation of satellite retrieval requirements', J. Atmos. Oceanic Technol., 28, 530-8. 
Gerber, H. (1996) 'Microphysics of marine stratocumulus clouds with two drizzle modes', J. Atmos. Sci., 53, 1649-62.

Gerber, H., Frick, G., Malinowski, S.P., Brenguier, J.L. and Burnet, F. (2005) 'Holes and entrainment in stratocumulus', J. Atmos. Sci., 62, 443-59.

Hansen, J.E. and Travis, L.D. (1974) 'Light scattering in planetary atmospheres', Space Science Reviews, 16, 527-610.

Kay, J., Hillman, B.R., Klein, S.A., Zhang, Y., Medeiros, B. and co-authors (2012) 'Exposing global cloud biases in the Community Atmosphere Model (CAM) using satellite observations and their corresponding instrument simulators', J. Climate, 25, 5190207.

King, M.D., Tsay, S.-C., Platnick, S., Wang, M. and Liou, K.-N. (1998) 'Cloud retrieval algorithms for MODIS: Optical thickness, effective particle radius, and thermodynamic phase', NASA (ed.), available online at http://modis-atmos.gsfc.nasa.gov/ reference_atbd.html.

King, N., Bower, K., Crosier, J. and Crawford, I. (2012) 'Evaluating MODIS cloud retrievals with in situ observations from VOCALS-REx', Atmospheric Chemistry \&6 Physics Discussions, 12, 23679-729.

Klein, S.A. and Hartmann, D.L. (1993) 'The seasonal cycle of low stratiform clouds', J. Climate, 6, 1587-606.

Kokhanovsky, A. (20040 'The depth of sunlight penetration in cloud fields for remote sensing', IEEE Geoscience and Remote Sensing Letters, 1, 242-5.

Kokhanovsky, A. and Rozanov, V.V. (2011) 'Droplet vertical sizing in warm clouds using passive optical measurements from a satellite', Atmos. Meas. Tech. Discuss., 4, 5597629.

Kubar, T.L., Hartmann, D.L. and Wood, R. (2009) 'Understanding the importance of microphysics and macrophysics for warm rain in marine low clouds: Part I: Satellite observations', J. Atmos. Sci., 66, 2953-72.

Lebsock, M.D. and L'Ecuyer, T.S. (2011) 'The retrieval of warm rain from CloudSat', J. Geophys. Res., 116, D20209.

Lebsock, M.D., Stephens, G.L. and Kummerow, C. (2008) 'Multisensor satellite observations of aerosol effects on warm clouds', J. Geophys. Res., 113, D15205, doi:10.1029/ 2008JD009876.

Liang, L., Di Girolamo, L. and Platnick, S. (2009) 'View-angle consistency in reflectance, optical thickness and spherical albedo of marine water-clouds over the northeastern Pacific through MISR-MODIS fusion', Geophys. Res. Lett., 36, L09811.

Marshak, A., Davis, A., Wiscombe, W. and Cahalan, R. (1995) 'Radiative smoothing in fractal clouds', J. Geophys. Res., 100, 26247-61.

Marshak, A., Platnick, S., Várnai, T., Wen, G. and Cahalan, R.F. (2006) 'Impact of threedimensional radiative effects on satellite retrievals of cloud droplet sizes', J. Geophys. Res., 111, D09207, doi:10.1029/2005JD006686.

Martin, G.M., Johnson, D.W. and Spice, A. (1994) 'The measurement and parameterization of effective radius of droplets in warm stratocumulus clouds', J. Atmos. Sci., 51, $1823-42$.

Minnis, P., Arduini, R.F., Young, D.F., Ayers, J.K., Albracht, B. and co-authors (2004) 'An examination of the impact of drizzle drops on satellite retrieved effective particle sizes', in Proceedings of the 14th International Conference on Clouds and Precipitation, Bologna, Italy.

Minnis, P., Sun-Mack, S., Chen, Y., Khaiyer, M.M., Yi, Y. and co-authors (2011) 'CERES Edition-2 cloud property retrievals using TRMM VIRS and terra and aqua MODIS data: Part II: Examples of average results and comparisons with other data', IEEE Trans. Geosci. Remote Sens., 49, 4401-30. 
Nakajima, T. and King, M.D. (1990) 'Determination of the optical thickness and effective particle radius of clouds from reflected solar radiation measurements: Part I: Theory', J. Atmos. Sci., 47, 1878-93.

Nakajima, T.Y., Suzuki, K. and Stephens, G.L. (2010a) 'Droplet growth in warm water clouds observed by the A-Train: Part I: Sensitivity analysis of the MODIS-derived cloud droplet sizes', J. Atmos. Sci., 67, 1884-96.

Nakajima, T.Y., Suzuki, K. and Stephens, G.L. (2010b) 'Droplet growth in warm water clouds observed by the A-Train: Part II: A multisensor view', J. Atmos. Sci., 67, 1897-907.

Oreopoulos, L. and Davies, R. (1998) 'Plane parallel albedo biases from satellite observations: Part I: Dependence on resolution and other factors', J. Climate, 11, 919-32.

Painemal, D. and Zuidema, P. (2011) 'Assessment of MODIS cloud effective radius and optical thickness retrievals over the Southeast Pacific with VOCALS-Rex in-situ measurements', J. Geophys. Res. 116, D24206, doi:10.1029/2011JD016155.

Pincus, R. and Evans, K.F. (2009) 'Computational cost and accuracy in calculating threedimensional radiative transfer: Results for new implementations of Monte Carlo and SHDOM', J. Atmos. Sci., 66, 3131-46.

Pincus, R., Platnick, S., Ackerman, G.S. and Hofmann, R.J.P. (2012) 'Reconciling simulated and observed views of clouds: MODIS, ISCCP, and the limits of instrument simulators', J. Climate, 25, 4699-720.

Platnick, S. (2000) 'Vertical photon transport in cloud remote sensing problems', J. Geophys. Res., 105, 22919-35.

Platnick, S., King, M.D., Ackerman, S.A., Menzel, W.P., Baum, B.A. and co-authors (2003) 'The MODIS cloud products: Algorithms and examples from Terra', IEEE Trans. Geosci. Remote Sens., 41, 459-73.

Prabhakara, C., Fraser, R.S., Dalu, G., Wu, M.-L.C., Curran, R.J. and co-authors (1988) 'Thin cirrus clouds: Seasonal distribution over oceans deduced from Nimbus-4 IRIS', J. Appl. Meteor. 27, 379-99.

Pruppacher, H. and Klett, J. (1997) Microphysics of Clouds and Precipitation, Dordrecht, The Netherlands, Kluwer Academic Publishers.

Quaas, J. and Boucher, O. (2005) 'Constraining the first aerosol indirect radiative forcing in the LMDZ GCM using POLDER and MODIS satellite data', Geophys. Res. Lett., 32, L17814.

Quaas, J., Ming, Y., Menon, S., Takemura, T., Wang, M. and co-authors (2009) 'Aerosol indirect effects: General circulation model intercomparison and evaluation with satellite data', Atmos. Chem. Phys. Discuss., 9, 12731-79.

Rosenfeld, D., Wang, H. and Rasch, P.J. (2012) 'The roles of cloud drop effective radius and LWP in determining rain properties in marine stratocumulus', Geophys. Res. Lett., 39, L13801.

Seethala, C. and Horváth, Á. (2010) 'Global assessment of AMSR-E and MODIS cloud liquid water path retrievals in warm oceanic clouds', J. Geophys. Res., 115, D13202.

Stevens, B., Ackerman, A.S., Albrecht, B.A., Brown, A.R., Chlond, A. and co-authors (2001) 'Simulations of trade wind cumuli under a strong inversion', J. Atmos. Sci., 58, 1870-91.

Stubenrauch, C., Rossow, W., Kinne, S., Ackerman, S., Cesana, G. and co-authors (2012) 'ASSESSMENT OF GLOBAL CLOUD DATASETS FROM SATELLITES: Project and database initiated by the GEWEX Radiation Panel', BAMS, doi:10.1175/BAMSD-12-00117.1.

Twomey, S. (1974) 'Pollution and the planetary albedo', Atmospheric Environment, 41, $120-5$. 
Várnai, T. and Davies, R. (1999) 'Effects of cloud heterogeneities on shortwave radiation: Comparison of cloud-top variability and internal heterogeneity', J. Atmos. Sci., 56, 4206-24.

Várnai, T. and Marshak, A. (2002) 'Observations of three-dimensional radiative effects that influence MODIS cloud optical thickness retrievals', J. Atmos. Sci., 59, 1607-18.

Walther, A. and Heidinger, A.K. (2012) 'Implementation of the Daytime Cloud Optical and Microphysical Properties algorithm (DCOMP) in PATMOS-x', Journal of Applied Meteorology and Climatology, 51, 1371-90.

Wang, L., Qu, J.J., Xiong, X., Hao, X., Xie, Y. and co-authors (2006) 'A new method for retrieving band 6 of Aqua MODIS', Geoscience and Remote Sensing Letters, IEEE, 3, 267-70.

Wiscombe, W.J. (1979) 'MIE scattering calculations, advances in technique and fast, vector-shaped computer codes', NCAR Tech. Note, TN-140+STR, 66.

Wood, R. and Hartmann, D.L. (2006) 'Spatial variability of liquid water path in marine low cloud: The importance of mesoscale cellular convection', J. Climate, 19, 1748-64.

Wood, R., Comstock, K.K., Bretherton, C.S., Cornish, C., Tomlinson, J. and co-authors (2008) 'Open cellular structure in marine stratocumulus sheets', J. Geophys. Res., 113, D12207.

Zhang, Z. (2013) 'On the sensitivity of cloud effective radius retrieval based on spectral method to bi-modal droplet size distribution: A semi-analytical model', J. Quant. Spectrosc. Radiat. Transfer, 129, 79-88.

Zhang, Z. and Platnick, S. (2011) 'An assessment of differences between cloud effective particle radius retrievals for marine water clouds from three MODIS spectral bands', Journal of Geophysical Research-Atmospheres, 116, doi:10.1029/2011JD016216.

Zhang, Z., Ackerman, A., Feingold, G., Platnick, S., Pincus, R. and co-authors (2012) 'Effects of cloud horizontal inhomogeneity and drizzle on remote sensing of cloud droplet effective radius: Case studies based on large-eddy simulations', J. Geophys. Res., doi:10.1029/2012JD017655.

Zhang, Z., Platnick, S., Yang, P., Heidinger, A.K. and Comstock, J.M. (2010) 'Effects of ice particle size vertical inhomogeneity on the passive remote sensing of ice clouds', J. Geophys. Res., 115, D17203.

Zhang, Z., Yang, P., Kattawar, G., Riedi, J., C.-Labonnote, L. and co-authors (2009) 'Influence of ice particle model on satellite ice cloud retrieval: Lessons learned from MODIS and POLDER cloud product comparison', Atmospheric Chemistry and Physics, 9, 7115-29.

Zhao, G. and Di Girolamo, L. (2006) 'Cloud fraction errors for trade wind cumuli from EOS-Terra instruments', Geophys. Res. Lett., 33, L20802.

Zinner, T., Wind, G., Platnick, S. and Ackerman, A.S. (2010) 'Testing remote sensing on artificial observations: Impact of drizzle and 3D cloud structure on effective radius retrievals', Atmos. Chem. Phys., 10, 1221-59. 